\title{
Triply Periodic Monopoles and Difference Modules on Elliptic Curves
}

\author{
Takuro MOCHIZUKI
}

Research Institute for Mathematical Sciences, Kyoto University, Kyoto, 606-8502, Japan

E-mail: takuro@kurims.kyoto-u.ac.jp

Received October 29, 2019, in final form May 18, 2020; Published online June 03, 2020

https://doi.org/10.3842/SIGMA.2020.048

\begin{abstract}
We explain the correspondences between twisted monopoles with Dirac type singularity and polystable twisted mini-holomorphic bundles with Dirac type singularity on a 3-dimensional torus. We also explain that they are equivalent to polystable parabolic twisted difference modules on elliptic curves.
\end{abstract}

Key words: twisted monopoles; twisted difference modules; twisted mini-holomorphic bundles; Kobayashi-Hitchin correspondence

2020 Mathematics Subject Classification: 53C07; 58E15; 14D21; 81T13

\section{Introduction}

We studied the Kobayashi-Hitchin correspondences for singular monopoles with periodicity in one direction [4] or two directions [5]. In this paper, we study singular monopoles with periodicity in three directions. In the analytic aspect, this case is much simpler than the other cases because a 3-dimensional torus is compact. But, there still exist interesting correspondences with algebrogeometric objects. Moreover, everything is generalized to the twisted case. (See Section 2 for the twisted objects.) Though we also study a generalization to the twisted case, this introduction is devoted to explain the results in the untwisted case.

\subsection{Triply periodic monopoles with Dirac type singularity}

Let $Y$ be an oriented 3-dimensional $\mathbb{R}$-vector space with an Euclidean metric $g_{Y}$. Let $\Gamma$ be a lattice of $Y$. We set $\mathcal{M}:=Y / \Gamma$, which is equipped with the induced metric $g_{\mathcal{M}}$. Let $Z$ be a finite subset of $\mathcal{M}$. Let $E$ be a $C^{\infty}$-vector bundle on $\mathcal{M} \backslash Z$ with a Hermitian metric $h$, a unitary connection $\nabla$ and an anti-self-adjoint endomorphism $\phi$. A tuple $(E, h, \nabla, \phi)$ is called a monopole on $\mathcal{M} \backslash Z$ if the Bogomolny equation

$$
F(\nabla)=* \nabla \phi
$$

is satisfied, where $F(\nabla)$ denotes the curvature of $\nabla$, and $*$ denotes the Hodge star operator with respect to $g_{\mathcal{M}}$. A point of $P \in Z$ is called a Dirac type singularity of the monopole $(E, h, \nabla, \phi)$ if $\left|\phi_{Q}\right|_{h}=O\left(d(Q, Z)^{-1}\right)$ for any $Q \in \mathcal{M} \backslash Z$, where $\phi_{Q}$ denotes the element of the fiber $\operatorname{End}(E)_{\mid Q}$ over $Q$ induced by $\phi$, and $d(Q, Z)$ denotes the distance between $Q$ and $Z$. Note that the notion of Dirac type singularity was originally introduced by Kronheimer [3]. The above condition is equivalent to the original definition, according to [6].

This paper is a contribution to the Special Issue on Integrability, Geometry, Moduli in honor of Motohico Mulase for his 65th birthday. The full collection is available at https://www.emis.de/journals/SIGMA/Mulase.html 


\subsection{Mini-holomorphic bundles with Dirac type singularity}

Let us explain a correspondence between monopoles with Dirac type singularity and polystable mini-holomorphic bundles with Dirac type singularity on a 3-dimensional torus. (See Section 2 below for more details on the notions of mini-complex structures and mini-holomorphic bundles with Dirac type singularity on 3-dimensional manifolds.) It was formulated by Kontsevich and Soibelman [2].

\subsubsection{Mini-complex structure}

We take a linear coordinate system $\left(x_{1}, x_{2}, x_{3}\right)$ on $Y$ compatible with the orientation such that $g_{Y}=\sum d x_{i} d x_{i}$, and we set $t:=x_{1}$ and $w=x_{2}+\sqrt{-1} x_{3}$. The coordinate system induces a mini-complex structure on $\mathcal{M} \backslash Z$. A $C^{\infty}$-function $f$ on an open subset of $\mathcal{M}$ is called miniholomorphic if $\partial_{t} f=\partial_{\bar{w}} f=0$. Let $\mathcal{O}_{\mathcal{M} \backslash Z}$ denote the sheaf of mini-holomorphic functions on $\mathcal{M} \backslash Z$.

\subsubsection{Mini-holomorphic bundles with Dirac type singularity}

Let $\mathcal{V}$ be a locally free $\mathcal{O}_{\mathcal{M} \backslash Z}$-module. Let $P$ be a point of $Z$. We take a lift $\left(t_{0}, w_{0}\right) \in Y$ of $P$. Let $\epsilon$ and $\delta$ denote small positive numbers. Set $B_{w_{0}}(\delta):=\left\{w \in \mathbb{C}|| w-w_{0} \mid<\delta\right\}$ and $B_{w_{0}}^{*}(\delta):=\left\{w \in \mathbb{C}|0<| w-w_{0} \mid<\delta\right\}$. For any $t_{0}-\epsilon<t<t_{0}+\epsilon$, the restriction $\mathcal{V}_{\mid\{t\} \times B_{w_{0}}^{*}(\delta)}$ is naturally a locally free $\mathcal{O}_{B_{w_{0}}^{*}(\delta)}$-module. If $t \neq t_{0}$, they extend to locally free $\mathcal{O}_{B_{w_{0}}(\delta)}$-modules

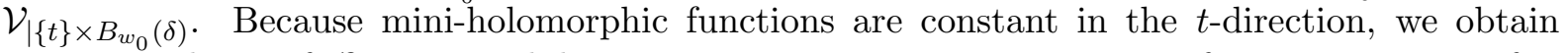
an isomorphism of $\mathcal{O}_{B_{w_{0}}^{*}(\delta)}$-modules $\mathcal{V}_{\mid\left\{t_{0}-\epsilon_{1}\right\} \times B_{w_{0}}^{*}(\delta)} \simeq \mathcal{V}_{\mid\left\{t_{0}+\epsilon_{1}\right\} \times B_{w_{0}}^{*}(\delta)}$ for $0<\epsilon_{1}<\epsilon$. If it is meromorphic at $w_{0}$, then $P$ is called a Dirac type singularity of $\mathcal{V}$. If every point of $Z$ is Dirac type singularity, then $\mathcal{V}$ is called a mini-holomorphic bundle with Dirac type singularity on $(\mathcal{M} ; Z)$.

\subsubsection{Stability condition}

Kontsevich and Soibelman [2] introduced a sophisticated way to define a stability condition for mini-holomorphic bundles with Dirac type singularity on $(\mathcal{M} ; Z)$.

Let $H^{j}(\mathcal{M} \backslash Z)$ denote the $j$-th cohomology group of $\mathcal{M} \backslash Z$ with $\mathbb{R}$-coefficient. Let $H_{j}(\mathcal{M}, Z)$ denote the relative $j$-th homology group of $(\mathcal{M}, Z)$ with $\mathbb{R}$-coefficient. Note that there exists the natural isomorphism

$$
\Phi_{Z}: H^{2}(\mathcal{M} \backslash Z) \simeq H_{1}(\mathcal{M}, Z) .
$$

Let $\mathfrak{T}$ denote the space of left invariant vector fields on $\mathcal{M}$, and let $\mathfrak{T}^{\vee}$ denote the left invariant 1 -forms on $\mathcal{M}$. Let $\sigma$ denote the image of 1 via the canonical morphism $\mathbb{R} \longrightarrow \mathfrak{T} \otimes \mathfrak{T}^{\vee}$. It is described as $\sigma=\sum_{i=1,2,3} \partial_{x_{i}} \otimes d x_{i}$.

For any mini-holomorphic bundle with Dirac type singularity $\mathcal{V}$ on $(\mathcal{M} ; Z)$, we obtain $c_{1}(\mathcal{V}) \in$ $H^{2}(\mathcal{M} \backslash Z)$, and hence $\Phi_{Z}\left(c_{1}(\mathcal{V})\right) \in H_{1}(\mathcal{M}, Z)$. Then, we obtain the following invariant vector field

$$
\int_{\Phi_{Z}\left(c_{1}(\mathcal{V})\right)} \sigma=\sum_{i=1,2,3}\left(\int_{\Phi_{Z}\left(c_{1}(\mathcal{V})\right)} d x_{i}\right) \partial_{x_{i}} \in \mathfrak{T} .
$$

Kontsevich and Soibelman discovered that $\int_{\Phi_{Z}\left(c_{1}(\mathcal{V})\right)} \sigma$ is a scalar multiplication of $\partial_{t}=\partial_{x_{1}}$, and they define the degree $\operatorname{deg}^{\mathrm{KS}}(\mathcal{V})$ for $\mathcal{V}$ as follows

$$
\int_{\Phi_{Z}\left(c_{1}(\mathcal{V})\right)} \sigma=\operatorname{deg}^{\mathrm{KS}}(\mathcal{V}) \partial_{t}
$$


They introduced the following stability condition.

Definition 1.1. A mini-holomorphic bundle with Dirac type singularity $\mathcal{V}$ on $(\mathcal{M} ; Z)$ is called stable (resp. semistable) if

$$
\begin{aligned}
& \operatorname{deg}^{\mathrm{KS}}\left(\mathcal{V}^{\prime}\right) / \operatorname{rank}\left(\mathcal{V}^{\prime}\right)<\operatorname{deg}^{\mathrm{KS}}(\mathcal{V}) / \operatorname{rank}(\mathcal{V}) \\
& \left(\operatorname{resp} . \operatorname{deg}^{\mathrm{KS}}\left(\mathcal{V}^{\prime}\right) / \operatorname{rank}\left(\mathcal{V}^{\prime}\right) \leq \operatorname{deg}^{\mathrm{KS}}(\mathcal{V}) / \operatorname{rank}(\mathcal{V})\right)
\end{aligned}
$$

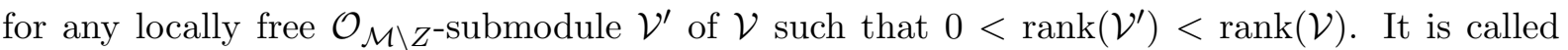
polystable if it is semistable and a direct sum of stable submodules.

\subsubsection{Kobayashi-Hitchin correspondence}

Let $(E, h, \nabla, \phi)$ be a monopole with Dirac type singularity on $\mathcal{M} \backslash Z$. We set $\partial_{E, \bar{w}}:=\nabla_{\bar{w}}$ and $\partial_{E, \partial_{t}}:=\nabla_{t}-\sqrt{-1} \phi$. Let $\mathcal{V}$ be the sheaf of sections $s$ of $E$ such that $\partial_{E, \bar{w}} s=\partial_{E, t} s=0$. It is a standard fact that $\mathcal{V}$ is a mini-holomorphic bundle with Dirac type singularity on $(\mathcal{M} ; Z)$. The following theorem was formulated by Kontsevich and Soibelman [2].

Theorem 1.2 (the untwisted case in Theorem 3.16, Proposition 4.2). The procedure induces an equivalence between monopoles with Dirac type singularity on $\mathcal{M} \backslash Z$ and polystable miniholomorphic bundles with Dirac type singularity of degree 0 on $(\mathcal{M} ; Z)$.

We shall relate the degree of Kontsevich and Soibelman with the analytic degree defined in terms of Hermitian metrics (Proposition 4.2). Then, Theorem 1.2 follows from the fundamental theorem due to Simpson [7] as we shall explain in the proof of Theorem 3.16, which is an analogue of a result due to Charbonneau and Hurtubise [1] for singular monopoles on 3-dimensional manifolds obtained as the product of $S^{1}$ and a compact Riemann surface. See also the work of Yoshino [8] on the Kobayashi-Hitchin correspondence for monopoles with Dirac type singularity on mini-complex 3-dimensional manifolds.

\subsection{Parabolic difference modules on elliptic curves}

Let us give a complement on correspondences between mini-holomorphic bundles with Dirac type singularity on a 3-dimensional torus and parabolic difference modules on elliptic curves.

Remark 1.3. After completing the first version of this paper, the author was informed that [2] also already contains the correspondence with difference modules on elliptic curves.

\subsubsection{Parabolic difference modules on elliptic curves and a stability condition}

Let $\Gamma_{0}$ be a lattice of $\mathbb{C}$. We set $T:=\mathbb{C} / \Gamma_{0}$. Let $\mathfrak{a} \in \mathbb{C}$. Let $\Phi: T \longrightarrow T$ be the morphism induced by $\Phi(z)=z+\mathfrak{a}$. Let $D \subset T$ be a finite subset. Let $\mathcal{O}_{T}(* D)$ denote the sheaf of meromorphic functions on $T$ which may have poles along $D$. For any $\mathcal{O}_{T}$-module $\mathcal{F}$, we set $\mathcal{F}(* D):=\mathcal{F} \otimes_{\mathcal{O}_{T}} \mathcal{O}_{T}(* D)$. A parabolic a-difference module on $T$ consists of the following data $V_{*}=\left(V,\left(\boldsymbol{\tau}_{P}, \mathcal{L}_{P}\right)_{P \in D}\right):$

- A locally free $\mathcal{O}_{T}$-module $V$.

- An isomorphism of $\mathcal{O}_{T}(* D)$-modules $V(* D) \simeq\left(\Phi^{*}\right)^{-1}(V)(* D)$.

- A sequence $0 \leq \tau_{P, 1}<\tau_{P, 2}<\cdots<\tau_{P, m(P)}<1$ for each $P \in D$.

- Lattices $\mathcal{L}_{P, i}(i=1, \ldots, m(P)-1)$ of the stalk $V(* D)_{P}$ at each $P \in D$. We formally set $\mathcal{L}_{P, 0}:=V_{P}$ and $\mathcal{L}_{P, m(P)}:=\left(\Phi^{*}\right)^{-1}(V)_{P}$ at each $P \in D$.

When we fix $\left(\boldsymbol{\tau}_{P}\right)_{P \in D}$, it is called a parabolic $\mathfrak{a}$-difference module on $\left(T,\left(\boldsymbol{\tau}_{P}\right)_{P \in D}\right)$. 
The degree of a parabolic a-difference module $\left(V,\left(\boldsymbol{\tau}_{P}, \mathcal{L}_{P}\right)_{P \in D}\right)$ is defined as follows

$$
\operatorname{deg}\left(V,\left(\boldsymbol{\tau}_{P}, \mathcal{L}_{P}\right)_{P \in D}\right):=\operatorname{deg}(V)+\sum_{P \in D} \sum_{i=1}^{m(P)}\left(1-\tau_{P, i}\right) \operatorname{deg}\left(\mathcal{L}_{P, i}, \mathcal{L}_{P, i-1}\right) .
$$

Here, we set $\operatorname{deg}\left(\mathcal{L}_{P, i}, \mathcal{L}_{P, i-1}\right):=\operatorname{length}\left(\mathcal{L}_{P, i} / \mathcal{L}_{P, i-1} \cap \mathcal{L}_{P, i}\right)-\operatorname{length}\left(\mathcal{L}_{P, i-1} / \mathcal{L}_{P, i-1} \cap \mathcal{L}_{P, i}\right)$. The degree can be rewritten as

$$
\operatorname{deg}\left(V,\left(\boldsymbol{\tau}_{P}, \mathcal{L}_{P}\right)_{P \in D}\right):=\operatorname{deg}(V)-\sum_{P \in D} \sum_{i=1}^{m(P)} \tau_{P, i} \operatorname{deg}\left(\mathcal{L}_{P, i}, \mathcal{L}_{P, i-1}\right),
$$

because $\sum_{P \in D} \sum_{i=1}^{m(P)} \operatorname{deg}\left(\mathcal{L}_{P, i}, \mathcal{L}_{P, i-1}\right)=0$. The slope is defined in the standard way

$$
\mu\left(V,\left(\boldsymbol{\tau}_{P}, \mathcal{L}_{P}\right)_{P \in D}\right):=\operatorname{deg}\left(V,\left(\boldsymbol{\tau}_{P}, \mathcal{L}_{P}\right)_{P \in D}\right) / \operatorname{rank} V .
$$

For any $\mathcal{O}_{T}(* D)$-submodule $0 \neq V^{\prime} \subset V$ such that $V^{\prime}(* D) \simeq\left(\Phi^{*}\right)^{-1}\left(V^{\prime}\right)(* D)$, we obtain lattices $\mathcal{L}_{P, i}^{\prime}$ of $V^{\prime}(* D)_{P}$ by setting $\mathcal{L}_{P, i}^{\prime}:=\mathcal{L}_{P, i} \cap V^{\prime}(* D)_{P}$ in $V(* D)_{P}$, and we obtain a parabolic $\mathfrak{a}$-difference module $\left(V^{\prime},\left(\boldsymbol{\tau}_{P}, \mathcal{L}_{P}^{\prime}\right)_{P \in D}\right)$. Such $\left(V^{\prime},\left(\boldsymbol{\tau}_{P}, \mathcal{L}_{P}^{\prime}\right)_{P \in D}\right)$ is called a parabolic a-difference submodule of $\left(V,\left(\boldsymbol{\tau}_{P}, \mathcal{L}_{P}\right)_{P \in D}\right)$.

Definition 1.4. $\left(V,\left(\boldsymbol{\tau}_{P}, \mathcal{L}_{P}\right)_{P \in D}\right)$ is called stable (resp. semistable) if

$$
\begin{aligned}
& \mu\left(V^{\prime},\left(\boldsymbol{\tau}_{P}, \mathcal{L}_{P}^{\prime}\right)_{P \in D}\right)<\mu\left(V,\left(\boldsymbol{\tau}_{P}, \mathcal{L}_{P}\right)_{P \in D}\right) \\
& \left(\operatorname{resp.} \mu\left(V^{\prime},\left(\boldsymbol{\tau}_{P}, \mathcal{L}_{P}^{\prime}\right)_{P \in D}\right) \leq \mu\left(V,\left(\boldsymbol{\tau}_{P}, \mathcal{L}_{P}\right)_{P \in D}\right)\right)
\end{aligned}
$$

for any parabolic a-difference submodules such that $0<\operatorname{rank} V^{\prime}<\operatorname{rank} V$. It is called polystable if it is semistable and a direct sum of stable objects.

\subsubsection{Equivalence}

We return to the situation in Section 1.2. We take a generator $e_{i}=\left(a_{i}, \alpha_{i}\right)(i=1,2,3)$ of $\Gamma \subset \mathbb{R}_{t} \times \mathbb{C}_{w}=Y$, which is compatible with the orientation of $Y$. We also assume that $\alpha_{1}$ and $\alpha_{2}$ generate a lattice in $\mathbb{C}$ and compatible with the orientation of $\mathbb{C}$. Let $\Gamma_{0}$ denote the lattice, and we set $T:=\mathbb{C} / \Gamma_{0}$. We set

$$
\gamma:=-\frac{a_{1} \bar{\alpha}_{2}-a_{2} \bar{\alpha}_{1}}{\alpha_{1} \bar{\alpha}_{2}-\alpha_{2} \bar{\alpha}_{1}}, \quad \mathfrak{t}:=a_{3}+2 \operatorname{Re}\left(\gamma \alpha_{3}\right), \quad \mathfrak{a}:=\alpha_{3} .
$$

It is easy to see that $\mathfrak{t}>0$. We define the isomorphism $F: \mathbb{R}_{t} \times \mathbb{C}_{w} \simeq \mathbb{R}_{s} \times \mathbb{C}_{u}$ by

$$
s=t+2 \operatorname{Re}(\gamma w), \quad u=w .
$$

Note that the induced action of $\Gamma$ on $\mathbb{R}_{s} \times \mathbb{C}_{u}$ is expressed as follows:

$$
e_{i}(s, u)=\left(s, u+\alpha_{i}\right) \quad(i=1,2), \quad e_{3}(s, u)=(s+\mathfrak{t}, u+\mathfrak{a}) .
$$

We set $\left[0, \mathfrak{t}\left[:=\{0 \leq s<\mathfrak{t}\}\right.\right.$. Let $Z_{Y}$ be the pull back of $Z$ by $Y \longrightarrow \mathcal{M}$. Let $D$ denote the image of the composite of the following maps:

$$
F\left(Z_{Y}\right) \cap\left(\left[0, \mathfrak{t}\left[\times \mathbb{C}_{u}\right) \subset \mathbb{R}_{s} \times \mathbb{C}_{u} \longrightarrow \mathbb{C}_{u} \longrightarrow T .\right.\right.
$$

For any $P \in D$, we take $u_{0} \in \mathbb{C}$ which is mapped to $P$. We obtain a sequence $0 \leq s_{P, 1}<$ $s_{P, 2}<\cdots<s_{P, m(P)}<\mathfrak{t}$ by the condition:

$$
\left\{\left(s_{P, i}, u_{0}\right) \mid i=1, \ldots, m(P)\right\}=F\left(Z_{Y}\right) \cap\left(\left[0, \mathfrak{t}\left[\times\left\{u_{0}\right\}\right) .\right.\right.
$$

It is independent of the choice of $u_{0}$. We set $\tau_{P, i}:=s_{P, i} / \mathfrak{t}$. 
Proposition 1.5 (the untwisted case in Propositions 3.13 and 3.14). There exists an equivalence between parabolic difference modules on $\left(T,\left(\boldsymbol{\tau}_{P}\right)_{P \in D}\right)$ and mini-holomorphic bundles with Dirac type singularity on $(\mathcal{M} ; Z)$. The equivalence preserves the degree up to the multiplication of a positive constant. As a result, the equivalence preserves the (poly)stability condition.

See Section 3.2.2 for the explicit correspondence. As a consequence of Theorem 1.2 and Proposition 1.5, we obtain the following theorem.

Theorem 1.6. We have the equivalence of the following objects:

- Monopoles with Dirac type singularity on $\mathcal{M} \backslash Z$.

- Polystable mini-holomorphic bundles with Dirac type singularity of degree 0 on $(\mathcal{M} ; Z)$.

- Polystable parabolic difference modules of degree 0 on $\left(T,\left(\boldsymbol{\tau}_{P}\right)_{P \in D}\right)$.

Here, $Z$ and $\left(\boldsymbol{\tau}_{P}\right)_{P \in D}$ are related as above.

This study is partially motivated by the holomorphic Floer theory [2] of Kontsevich and Soibelman. Among other things, they revisit the Riemann-Hilbert correspondence for $D$ modules from the viewpoint of symplectic topology, and they extend it to the context of difference modules of various types. Moreover, they propose an analogue of the non-abelian Hodge theory in the context of difference modules, where the role of harmonic bundles should be played by monopoles as in Theorem 1.6.

Though the untwisted case is explained in this introduction, we shall study the twisted case, i.e., equivalences of twisted mini-holomorphic bundles, twisted difference modules, and twisted monopoles. We should note that Kontsevich and Soibelman suggested that there should exist a twisted version of of Theorem 1.6.

\section{Preliminary}

We introduce the notions of twisted mini-holomorphic bundles and twisted monopoles as generalizations of the notions of mini-holomorphic bundles [4] and monopoles. We are interested only in the case where the base manifolds are 3-dimensional torus. We also introduce twisted difference modules on elliptic curves.

\subsection{Mini-complex structure on 3-dimensional manifolds}

Let $(t, w)$ denote the standard coordinate system on $\mathbb{R} \times \mathbb{C}$. Let $M$ be an oriented 3-dimensional $C^{\infty}$-manifold. A mini-complex coordinate system on $M$ is a family of open subsets $U_{\lambda}(\lambda \in \Lambda)$ equipped with an oriented embedding $\varphi_{\lambda}: U_{\lambda} \longrightarrow \mathbb{R} \times \mathbb{C}$ satisfying the following conditions.

- $M=\bigcup_{\lambda \in \Lambda} U_{\lambda}$.

- Let $F_{\lambda, \mu}: \varphi_{\mu}\left(U_{\lambda} \cap U_{\mu}\right) \longrightarrow \varphi_{\lambda}\left(U_{\lambda} \cap U_{\mu}\right)$ denote the induced diffeomorphism of open subsets in $\mathbb{R} \times \mathbb{C}$. Note that $F_{\lambda, \mu}$ is expressed as $\left(\left(F_{\lambda, \mu}\right)_{t}(t, w),\left(F_{\lambda, \mu}\right)_{w}(t, w)\right)$ in terms of the coordinate systems. Then, it holds that $\partial_{t}\left(F_{\lambda, \mu}\right)_{w}=0$ and $\partial_{\bar{w}}\left(F_{\lambda, \mu}\right)_{w}=0$.

Two mini-complex coordinate systems $\left\{\left(U_{\lambda}, \varphi_{\lambda}\right)\right\}_{\lambda \in \Lambda}$ and $\left\{\left(V_{\mu}, \psi_{\mu}\right)\right\}_{\mu \in \Gamma}$ are called equivalent if their union is also a mini-complex coordinate system. A mini-complex structure on $M$ is an equivalence class of mini-complex coordinate systems. We shall not distinguish a mini-complex structure and a mini-complex coordinate system contained in the mini-complex structure.

Suppose that $M$ is equipped with a mini-complex structure. On a mini-complex coordinate neighbourhood $(U ; t, w)$, let $T_{S} U$ denote the subbundle of the tangent bundle $T U$ generated 
by $\partial_{t}$. By patching $T_{S} U$ for any mini-complex coordinate neighbourhoods $(U ; t, w)$ we obtain the subbundle $T_{S} M \subset T M$.

Let $T_{S}^{*} M$ denote the dual bundle of $T_{S} M$. Let $T_{Q}^{*} M$ denote the kernel of the natural surjection $T^{*} M \longrightarrow T_{S}^{*} M$. It is naturally equipped with a complex structure $J$. Let $\Omega_{Q}^{1,0} M \subset T_{Q}^{*} M \otimes \mathbb{C}$ (resp. $\Omega_{Q}^{0,1} M$ ) denote the eigen subbundle with respect to $J$ corresponding to $\sqrt{-1}$ (resp. $-\sqrt{-1}$ ). We set $\Omega^{0,1} M:=\left(T^{*} M \otimes \mathbb{C}\right) / \Omega_{Q}^{1,0} M$ and $\Omega^{0, i} M:=\bigwedge^{i} \Omega^{0,1} M$ for $i=0,1,2$. Similarly, we set $\Omega^{1,0} M:=\left(T^{*} M \otimes \mathbb{C}\right) / \Omega_{Q}^{0,1} M$ and $\Omega^{i, 0} M:=\bigwedge^{i} \Omega^{1,0} M$ for $i=0,1,2$.

Let $\bar{\partial}_{M}$ denote the differential operator $C^{\infty}(M, \mathbb{C}) \longrightarrow C^{\infty}\left(M, \Omega^{0,1} M\right)$ induced by the exterior derivative and the projection $T^{*} M \otimes \mathbb{C} \longrightarrow \Omega^{0,1} M$. The induced operator

$$
C^{\infty}\left(M, \Omega^{0,1} M\right) \longrightarrow C^{\infty}\left(M, \Omega^{0,2} M\right)
$$

is also denoted by $\bar{\partial}_{M}$. Similarly, we obtain the operator

$$
\partial_{M}: C^{\infty}\left(M, \Omega^{i, 0} M\right) \longrightarrow C^{\infty}\left(M, \Omega^{i+1,0} M\right) .
$$

\subsubsection{Riemannian case}

Suppose that $M$ is also equipped with a Riemannian metric $g_{M}$. Let $T_{S, g_{M}}^{*} M$ denote the orthogonal complement of $T_{Q}^{*} M$. We shall naturally identify $T_{S, g_{M}}^{*} M$ and $T_{S}^{*} M$.

Because $T^{*} M$ and $T_{Q}^{*} M$ are oriented, $T_{S, g_{M}}^{*} M$ is also oriented. Let $\eta$ be the unique section of $T_{S, g_{M}}^{*} M$ in the positive direction such that the norm of $\eta$ is 1 . By $\eta, T_{S, g_{M}}^{*} M$ is identified with $\mathbb{R} \times M$. If there exists a mini-complex coordinate system $(U ; t, w)$ such that $g_{M \mid U}=$ $d t d t+d w d \bar{w}$, then $\eta_{\mid M}=d t$.

We obtain a decomposition

$$
T^{*} M \otimes \mathbb{C}=\Omega_{Q}^{1,0} M \oplus \Omega_{Q}^{0,1} M \oplus T_{S, g_{M}}^{*} M \otimes \mathbb{C} .
$$

We also obtain the isomorphisms

$$
\Omega_{Q}^{1,0} M \oplus T_{S, g_{M}}^{*} M \otimes \mathbb{C} \simeq \Omega^{1,0} M, \quad \Omega_{Q}^{0,1} M \oplus T_{S, g_{M}}^{*} M \otimes \mathbb{C} \simeq \Omega^{0,1} M .
$$

If the complex structure $J$ on $T_{Q}^{*} M$ is an isometry with respect to $g_{M}$, the decomposition (2.1) is orthogonal.

\subsection{Twisted mini-holomorphic bundles}

Let $M$ be a mini-complex 3-dimensional manifold. Let $E$ be a $C^{\infty}$-vector bundle on $M$. We shall always assume that the rank of $E$ is finite. Let $\varrho \in C^{\infty}\left(M, \Omega^{0,2} M\right)$.

Definition 2.1. A $\varrho$-twisted mini-holomorphic structure of $E$ is a differential operator $\bar{\partial}_{E}$ : $C^{\infty}(M, E) \longrightarrow C^{\infty}\left(M, \Omega^{0,1} M \otimes E\right)$ such that the following conditions are satisfied.

- $\bar{\partial}_{E}(f s)=f \bar{\partial}_{E}(s)+\left(\bar{\partial}_{M} f\right) \otimes s$ holds for any $f \in C^{\infty}(M, \mathbb{C})$ and $s \in C^{\infty}(M, E)$.

- The induced operator $C^{\infty}\left(M, \Omega^{0,1} M \otimes E\right) \longrightarrow C^{\infty}\left(M, \Omega^{0,2} M \otimes E\right)$ is also denoted by $\bar{\partial}_{E}$. Then, $\bar{\partial}_{E} \circ \bar{\partial}_{E}=\varrho \operatorname{id}_{E}$ holds.

Such $\left(E, \bar{\partial}_{E}\right)$ is called a $\varrho$-twisted mini-holomorphic vector bundle. If $\varrho=0$, we shall omit the adjective "0-twisted". 
Remark 2.2. A $C^{\infty}$-function $f$ on an open subset $\mathcal{U} \subset M$ is called mini-holomorphic if $\bar{\partial}_{M} f=0$. Let $\mathcal{O}_{M}$ denote the sheaf of mini-holomorphic functions. In the case $\varrho=0$, miniholomorphic bundles are naturally identified with locally free $\mathcal{O}_{M}$-modules of finite rank. Let $\left(E, \bar{\partial}_{E}\right)$ be a mini-holomorphic bundle on $M$. A local section $s$ of $E$ is called mini-holomorphic if $\bar{\partial}_{E}(s)=0$. Let $\widetilde{E}$ denote the sheaf of mini-holomorphic sections of $E$. Then, it is easy to observe that $\widetilde{E}$ is a locally free $\mathcal{O}_{M}$-module of finite rank. This correspondence induces an equivalence between mini-holomorphic bundles and locally free $\mathcal{O}_{M}$-modules of finite rank.

\subsubsection{Scattering map}

Let $\left(E, \bar{\partial}_{E}\right)$ be a $\varrho$-twisted mini-holomorphic vector bundle on $M$. Let $\gamma:[0,1] \longrightarrow M$ be a $C^{\infty}$ path such that $T \gamma(T[0,1]) \subset T_{S} M$. Then, $\gamma^{-1}(E)$ is equipped with a connection induced by the $\varrho$-twisted mini-holomorphic structure $\bar{\partial}_{E}$, and hence we obtain the induced isomorphism $E_{\gamma(0)} \simeq E_{\gamma(1)}$. It is called the scattering map in [1].

Let $(U ; t, w)$ be a mini-complex coordinate neighbourhood of $M$. Let $\partial_{E, t}\left(\right.$ resp. $\left.\partial_{E, \bar{w}}\right)$ denote the differential operators of $E_{\mid U}$ induced by $\bar{\partial}_{E}$ and $\partial_{t}$ (resp. $\partial_{\bar{w}}$ ). We have the expression $\varrho=\varrho_{0} d t d \bar{w}$. Then, the condition $\bar{\partial}_{E} \circ \bar{\partial}_{E}=\varrho \operatorname{id}_{E}$ on $U$ is equivalent to $\left[\partial_{E, t}, \partial_{E, \bar{w}}\right]=\varrho_{0} \mathrm{id}_{E}$. Assume that there exists $\nu=\nu_{t} d t+\nu_{\bar{w}} d \bar{w} \in C^{\infty}\left(U, \Omega^{0,1}\right)$ such that $\bar{\partial} \nu=\varrho$ on $U$. Note that such $\nu$ always exists locally. On $U$, we set $\bar{\partial}_{E}^{\nu}=\bar{\partial}_{E}-\nu \operatorname{id}_{E}$. Then, $\left(E_{\mid U}, \bar{\partial}_{E}^{\nu}\right)$ is clearly a mini-holomorphic bundle.

Suppose that $U$ is isomorphic to $\left\{t_{0}<t<t_{1}\right\} \times B_{\delta}$, where $B_{\delta}=\{w \in \mathbb{C}|| w \mid<\delta\}$. Take $t_{0}<b_{1}<b_{2}<t_{1}$. We obtain the scattering map $F: E_{\mid\left\{t=b_{1}\right\} \times B_{\delta}} \simeq E_{\mid\left\{t=b_{2}\right\} \times B_{\delta}}$. Let $\partial_{E, \bar{w}, b_{i}}$ denote the operators on $E_{\mid\left\{t=b_{i}\right\} \times B_{\delta}}$ by $\partial_{E, \bar{w}}$.

Lemma 2.3. $F^{*}\left(\partial_{E, \bar{w}, b_{2}}\right)=\partial_{E, \bar{w}, b_{1}}+\left(\int_{b_{1}}^{b_{2}} \varrho_{0} d t\right) \mathrm{id}$.

Proof. Take $\nu=\nu \bar{w} d \bar{w}$ such that $\bar{\partial} \nu=\varrho$, i.e., $\partial_{t} \nu_{\bar{w}}=\varrho_{0}$. Then, $F^{*}\left(\partial_{E, \bar{w}, b_{2}}^{\nu}\right)=\partial_{E, \bar{w}, b_{1}}^{\nu}$ because of $\left[\partial_{E, t}^{\nu}, \partial_{E, \bar{w}}^{\nu}\right]=0$. Then, the claim of the lemma follows.

\subsubsection{Twisted mini-holomorphic bundles with Dirac type singularity}

Let $Z \subset M$ be a discrete subset. Let $\left(E, \bar{\partial}_{E}\right)$ be a $\varrho$-twisted mini-holomorphic bundle on $M \backslash Z$. Let $P$ be a point of $Z$. Let $(U ; t, w)$ be a mini-complex coordinate neighbourhood around $P \in Z$. We may assume $(t(P), w(P))=(0,0)$. By shrinking $U$, we assume that $U \simeq\{-2 \epsilon<t<2 \epsilon\} \times B_{\delta}$ by the mini-complex coordinate system for some $\epsilon>0$ and $\delta>0$. Set $B_{\delta}^{*}:=B_{\delta} \backslash\{0\}$. We obtain the scattering map $F: E_{\mid\{-\epsilon\} \times B_{\delta}^{*}} \simeq E_{\mid\{\epsilon\} \times B_{\delta}^{*}}$.

Definition 2.4. $P$ is a Dirac type singularity of $\left(E, \bar{\partial}_{E}\right)$ if $F$ and $F^{-1}$ are $O\left(|w|^{-N}\right)$ for some

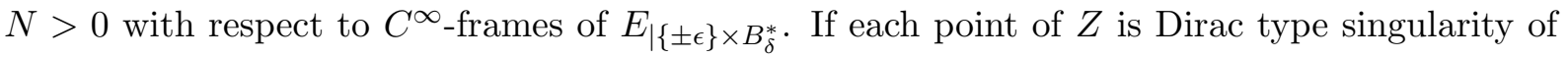
$\left(E, \bar{\partial}_{E}\right)$, we say that $\left(E, \bar{\partial}_{E}\right)$ is a $\varrho$-twisted mini-holomorphic bundle with Dirac type singularity on $(M ; Z)$.

Take $\nu=\nu_{t} d t+\nu_{\bar{w}} d \bar{w} \in C^{\infty}\left(U, \Omega^{0,1}\right)$ such that $\bar{\partial} \nu=\varrho$. We set $\bar{\partial}_{E}^{\nu}:=\bar{\partial}_{E_{\mid U}}-\nu$ id so that $\left(E_{\mid U}, \bar{\partial}_{E}^{\nu}\right)$ is mini-holomorphic. The scattering map $F^{\nu}: E_{\mid\{-\epsilon\} \times B_{\delta}^{*}} \simeq E_{\mid\{\epsilon\} \times B_{\delta}^{*}}$ for $\bar{\partial}_{E}^{\nu}$ is holomorphic with respect to $\partial_{E, \bar{w}}^{\nu}$. Note that $F^{\nu}=\exp \left(\int_{-\epsilon}^{\epsilon} \nu_{t}\right) F$. The condition in Definition 2.4 is satisfied if and only if $F^{\nu}$ extends to a meromorphic isomorphism $\left(E_{\mid\{-\epsilon\} \times B_{\delta}}, \partial_{E, \bar{w},-\epsilon}^{\nu}\right)(* 0) \simeq$ $\left(E_{\mid\{\epsilon\} \times B_{\delta}}, \partial_{E, \bar{w}, \epsilon}^{\nu}\right)(* 0)$, i.e., $P$ is Dirac type singularity of $\left(E_{\mid U}, \bar{\partial}_{E}^{\nu}\right)$ in the sense of [4, Section 2.2].

We regard $U$ as an open subset of $\mathbb{R} \times \mathbb{C}$ by the coordinate system $(t, w)$. Let $\varphi: \mathbb{C}^{2} \longrightarrow$ $\mathbb{R} \times \mathbb{C}$ be given by $\varphi\left(z_{1}, z_{2}\right)=\left(\left|z_{1}\right|^{2}-\left|z_{2}\right|^{2}, 2 z_{1} z_{2}\right)$. Let $\widetilde{U}$ be the pull back of $U$ by $\varphi$. The mini-holomorphic bundle $\left(E, \bar{\partial}_{E}^{\nu}\right)_{\mid U \backslash\{P\}}$ induces an $S^{1}$-equivariant holomorphic vector bundle 
$\left(\widetilde{E}_{P}^{\prime}, \bar{\partial}_{\widetilde{E}_{P}^{\prime}}^{\nu}\right)$ on $\widetilde{U} \backslash\{(0,0)\}$, which uniquely extends to an $S^{1}$-equivariant holomorphic vector bundle $\left(\widetilde{E}_{P}^{\nu}, \bar{\partial}_{\widetilde{E}_{P}}^{\nu}\right)$ on $\widetilde{U}$. (See $[6$, Section 2.2] for a more detailed explanation.)

Lemma 2.5. Suppose that $\nu_{i}=\nu_{i, t} d t+\nu_{i, \bar{w}} d \bar{w} \in C^{\infty}\left(U, \Omega^{0,1}\right)(i=1,2)$ satisfy $\bar{\partial} \nu_{i}=\varrho$. Then, the natural identification $\widetilde{E}_{P \mid \widetilde{U} \backslash\{(0,0)\}}^{\nu_{1}}=\widetilde{E}_{P}^{\prime}=\widetilde{E}_{P \mid \widetilde{U} \backslash\{(0,0)\}}^{\nu_{2}}$ uniquely extends to a $C^{\infty}$. isomorphism $\widetilde{E}_{P}^{\nu_{1}} \simeq \widetilde{E}_{P}^{\nu_{2}}$.

Proof. Set $\nu_{0}=\nu_{0, t} d t+\nu_{0, \bar{w}} d \bar{w}:=\nu_{2}-\nu_{1}$. We have $\bar{\partial}_{E}^{\nu_{2}}=\bar{\partial}_{E}^{\nu_{1}}-\nu_{0} \mathrm{id}_{E}$. By the construction (see [6, Section 2.2]), we have $\bar{\partial}_{\widetilde{E}_{P}^{\prime}}^{\nu_{2}}=\bar{\partial}_{\widetilde{E}_{P}^{\prime}}^{\nu_{1}}-\left(\varphi^{*}\left(\nu_{0, t}\right) \bar{\partial} \varphi^{*}(t)+\varphi^{*}\left(\nu_{0, \bar{w}}\right) \bar{\partial} \varphi^{*}(\bar{w})\right)$ id. Then, the claim of the lemma is clear.

We set $\widetilde{E}_{P}:=\widetilde{E}_{P}^{\nu}$ for $\nu \in C^{\infty}\left(U, \Omega^{0,1}\right)$ such that $\bar{\partial} \nu=\varrho$, which is called the Kronheimer resolution of $\left(E, \bar{\partial}_{E}\right)$ at $P$.

Definition 2.6. A Hermitian metric $h$ of $E$ is called adapted at $P$ if the induced metric $\widetilde{h}_{P}$ of $\widetilde{E}_{P}^{\prime}$ extends to a $C^{\infty}$-metric of the Kronheimer resolution $\widetilde{E}_{P}$. If $h$ is adapted at any point of $Z$, then $h$ is called an adapted metric of $\left(E, \bar{\partial}_{E}\right)$.

\subsubsection{Chern connections and Higgs fields}

Suppose that we are given a splitting $T M / T_{S} M \longrightarrow T M$. It induces the following decompositions:

$$
\begin{aligned}
& T^{*} M \otimes \mathbb{C} \simeq \Omega_{Q}^{1,0} M \oplus \Omega_{Q}^{0,1} M \oplus T_{S}^{*} M \otimes \mathbb{C}, \\
& \Omega^{0,1} M \simeq \Omega_{Q}^{0,1} M \oplus T_{S}^{*} M \otimes \mathbb{C} \\
& \Omega^{1,0} M \simeq \Omega_{Q}^{0,1} M \oplus T_{S}^{*} M \otimes \mathbb{C} .
\end{aligned}
$$

Let $\left(E, \bar{\partial}_{E}\right)$ be a $\varrho$-twisted mini-holomorphic bundle on $M$. By $(2.3)$, we obtain a decomposition $\bar{\partial}_{E}=\bar{\partial}_{E}^{S} \oplus \bar{\partial}_{E}^{Q}$, where $\bar{\partial}_{E}^{S}(s) \in C^{\infty}\left(X,\left(T_{S} M \otimes \mathbb{C}\right)^{\vee}\right)$ and $\bar{\partial}_{E}^{Q}(s) \in C^{\infty}\left(X, \Omega_{O}^{0,1} M\right)$.

Let $h$ be a Hermitian metric of $E$. We obtain the differential operator $\partial_{E, h}: C^{\infty}(X, E) \longrightarrow$ $C^{\infty}\left(X, \Omega^{1,0} M \otimes E\right)$ satisfying the condition $\bar{\partial}_{M} h(u, v)=h\left(\bar{\partial}_{E} u, v\right)+h\left(u, \partial_{E, h} v\right)$ for any $u, v \in$ $C^{\infty}(X, E)$. We also obtain the decomposition $\partial_{E, h}=\partial_{E, h}^{Q}+\partial_{E, h}^{S}$ induced by (2.4). For a minicomplex coordinate neighbourhood $(U ; t, w)$, we obtain the operators $\partial_{E, h, w}\left(\right.$ resp. $\left.\partial_{E, h, t}\right)$ on $E$ induced by $\partial_{E, h}$ and $\partial_{w}\left(\right.$ resp. $\left.\partial_{t}\right)$.

Remark 2.7. In [4], $\partial_{E, h, t}$ is denoted as $\partial_{E, h, t}^{\prime}$.

By using (2.2), we set

$$
\nabla_{h}:=\bar{\partial}_{E}^{Q}+\partial_{E, h}^{Q}+\frac{1}{2}\left(\bar{\partial}_{E}^{S}+\partial_{E, h}^{S}\right), \quad \phi_{h}:=\frac{\sqrt{-1}}{2}\left(\bar{\partial}_{E}^{S}-\partial_{E, h}^{S}\right) .
$$

They are called the Chern connection and the Higgs field of $\left(E, \bar{\partial}_{E}, h\right)$. Note that they depend on the choice of a splitting $T M / T_{S} M \longrightarrow T M$.

If $M$ is also equipped with a Riemannian metric $g_{M}$, we shall use the splitting $T M / T_{S} M \longrightarrow$ $T M$ induced by $g_{M}$. Moreover, by the section $\eta$ in Section 2.1.1, $T_{S, g_{M}}^{*} M$ is identified with the product bundle $\mathbb{R} \times M$. Hence, we regard $\phi_{h}$ as an anti-Hermitian endomorphism of $E$. In particular, if $g_{M}=d t d t+d w d \bar{w}$ on a mini-complex coordinate neighbourhood $(U ; t, w)$, the following holds for any $s \in C^{\infty}(U, E)$ :

$$
\begin{aligned}
& \nabla_{h}(s)=\left(\partial_{E, \bar{w}} s\right) d \bar{w}+\left(\partial_{E, h, w} s\right) d w+\frac{1}{2}\left(\partial_{E, t} s+\partial_{E, h, t} s\right) d t, \\
& \phi_{h}(s)=\frac{\sqrt{-1}}{2}\left(\partial_{E, t} s-\partial_{E, h, t} s\right) .
\end{aligned}
$$




\subsection{Twisted monopoles in the locally Euclidean case}

\subsubsection{Twisted monopoles}

Let $\left(M, g_{M}\right)$ be an oriented Riemannian 3-dimensional manifold. Let $B$ be a real 2-form on $M$. Let $E$ be a vector bundle on $M$ equipped with a Hermitian metric $h$, a unitary connection $\nabla$, and an anti-Hermitian endomorphism $\phi$.

Definition 2.8. Such a tuple $(E, h, \nabla, \phi)$ is called a $B$-twisted monopole if the following $B$ twisted Bogomolny equation is satisfied:

$$
F(\nabla)=* \nabla \phi+\sqrt{-1} B \mathrm{id}_{E} .
$$

Here $F(\nabla)$ denotes the curvature of $\nabla$, and $*$ denotes the Hodge star operator.

Let $A$ and $f$ be a real 1 -form and an $\mathbb{R}$-valued $C^{\infty}$-function on $M$, respectively. We set $\widetilde{\nabla}:=\nabla+\sqrt{-1} A$ id, $\widetilde{\phi}:=\phi+\sqrt{-1} f$ id and $\widetilde{B}:=B+d A-*(d f)$. Then, the following is easy to see.

Lemma 2.9. $(E, h, \nabla, \phi)$ is a B-twisted monopole if and only if $(E, h, \widetilde{\nabla}, \widetilde{\phi})$ is a $\widetilde{B}$-twisted monopole.

Remark 2.10. If $M$ is compact, any real 2-form $B$ on $M$ is expressed as $B=d A-* d f+B_{0}$, where $A$ is a real 1 -form, $f$ is a $\mathbb{R}$-valued $C^{\infty}$-function, and $B_{0}$ is a harmonic 1 -form. Indeed, let $G$ denote the Green operator for the Laplace-Beltrami operator on the space of 2-forms on $M$. Then, $B-\left(d^{*} d+d d^{*}\right) G(B)$ is a harmonic 2-form, and $G(B)$ is $C^{\infty}$. We can also deduce that for any point $P \in M$, there exists a neighbourhood $M_{P}$ of $P$ such that $B_{\mid M_{P}}=d A_{P}-* d f_{P}$ for a real 1-form $A_{P}$ and an $\mathbb{R}$-valued $C^{\infty}$-function $f_{P}$ on $M_{P}$.

\subsubsection{Twisted monopoles and twisted mini-holomorphic bundles in the locally Euclidean case}

Suppose that $M$ is also equipped with a mini-complex structure. Moreover, we assume that $M$ is a locally Euclidean, i.e., for each $P \in M$, there exists a mini-complex coordinate neighbourhood $(U ; t, w)$ of $P$ such that the Riemannian metric of $M$ on $U$ is $d t d t+d w d \bar{w}$. Note that $d \eta=0$ for the global trivialization $\eta$ of $T_{S, g_{M}}^{*} M$ in Section 2.1.1. By (2.1), for any complex vector bundle $\mathcal{V}$ on $M$, we obtain the decomposition

$$
\mathcal{V} \otimes \bigwedge^{2}\left(T^{*} M \otimes \mathbb{C}\right)=\left(\mathcal{V} \otimes \Omega_{Q}^{1,0} M \wedge \eta\right) \oplus\left(\mathcal{V} \otimes \Omega_{Q}^{0,1} M \wedge \eta\right) \oplus\left(\mathcal{V} \otimes \Omega_{Q}^{1,1} M\right)
$$

where $\Omega_{Q}^{1,1} M:=\Omega_{Q}^{1,0} M \wedge \Omega_{Q}^{0,1} M$. For any section $s$ of $\mathcal{V} \otimes \bigwedge^{2}\left(T^{*} M \otimes \mathbb{C}\right)$, we obtain the decomposition $s=s^{(1,0), \eta}+s^{(0,1), \eta}+s^{(1,1)}$ according to (2.5). In particular, we obtain the decomposition $B=B^{(1,0), \eta}+B^{(0,1), \eta}+B^{(1,1)}$. Because $B$ is real, $B^{(1,1)}$ is also real, and $B^{(1,0), \eta}=$ $B^{(0,1), \eta}$ holds.

We can check the following lemma by a direct computation.

Lemma 2.11. Let $(E, h, \nabla, \phi)$ be a B-twisted monopole on $M$. We have the decomposition $\nabla=\nabla_{Q}^{1,0}+\nabla_{Q}^{0,1}+\nabla_{S}$ induced by (2.1). We set $\bar{\partial}_{E}:=\nabla_{Q}^{0,1}+\nabla_{S}-\sqrt{-1} \phi \eta$. Then, $\left(E, \bar{\partial}_{E}\right)$ is a $\sqrt{-1} B^{(0,1), \eta}$-twisted mini-holomorphic bundle.

Conversely, let $\left(E, \bar{\partial}_{E}\right)$ be a $\varrho$-twisted mini-holomorphic bundle on $M$. Let $h$ be a Hermitian metric of $E$. We obtain the Chern connection $\nabla_{h}$ and the Higgs field $\phi_{h}$.

Lemma 2.12. We have $\left(F\left(\nabla_{h}\right)-* \nabla_{h} \phi_{h}\right)^{(0,1), \eta}=\varrho \operatorname{id}_{E}$ and $\left(F\left(\nabla_{h}\right)-* \nabla_{h} \phi_{h}\right)^{(1,0), \eta}=-\bar{\varrho} \operatorname{id}_{E}$. 
Proof. We have $\bar{\partial}_{E}=\nabla_{h, Q}^{0,1}+\nabla_{h, S}-\sqrt{-1} \phi_{h} \eta$ and $\partial_{E, h}=\nabla_{h, Q}^{1,0}+\nabla_{h, S}+\sqrt{-1} \phi_{h} \eta$. Because $\bar{\partial}_{E} \circ \bar{\partial}_{E}=\varrho$ id, we obtain $\partial_{E, h} \circ \partial_{E, h}=-\bar{\varrho}$ id. Then, we obtain the claim of the lemma by computations.

Corollary 2.13. There exists a real 2 -form $B$ such that $\left(E, h, \nabla_{h}, \phi_{h}\right)$ is a B-twisted monopole if and only if the trace-free part of $\left(F\left(\nabla_{h}\right)-* \nabla_{h} \phi_{h}\right)^{(1,1)}$ is 0, i.e., there exists a real 2-form $\varpi$ such that $\left(F\left(\nabla_{h}\right)-* \nabla_{h} \phi_{h}\right)^{(1,1)}=\sqrt{-1} \varpi \mathrm{id}$. In that case, $B=-\sqrt{-1}(\varrho-\bar{\varrho})+\varpi$.

Remark 2.14. If the condition in Corollary 2.13 is satisfied, $\left(E, \bar{\partial}_{E}, h\right)$ is also called a $B$-twisted monopole.

\subsubsection{Dirac type singularity}

Let $Z$ be a discrete subset of $M$. Let $B$ be a real 2 -form on $M$. Let $(E, h, \nabla, \phi)$ be a $B$-twisted monopole on $M \backslash Z$. Let $\left(E, \bar{\partial}_{E}\right)$ be the underlying $\sqrt{-1} B^{(0,1), \eta}$-twisted mini-holomorphic bundle.

Definition 2.15. A point $P \in Z$ is called Dirac type singularity of $(E, h, \nabla, \phi)$ if the following conditions are satisfied:

- $P$ is Dirac type singularity of $\left(E, \bar{\partial}_{E}\right)$.

- $h$ is an adapted metric of $\left(E, \bar{\partial}_{E}\right)$ in the sense of Definition 2.6.

We say that $(E, h, \nabla, \phi)$ is a $B$-twisted monopole with Dirac type singularity on $(M ; Z)$ if any point $P \in Z$ is Dirac type singularity of $(E, h, \nabla, \phi)$.

Lemma 2.16. $P$ is Dirac type singularity of $(E, h, \nabla, \phi)$ if and only if there exists a neighbourhood $M_{P}$ of $P$ in $M$ such that $\left|\phi_{Q}\right|_{h}=O\left(d(P, Q)^{-1}\right)$ for $Q \in M_{P} \backslash\{P\}$.

Proof. If $M_{P}$ is sufficiently small, there exists a real 1-form $A_{P}$ and an $\mathbb{R}$-valued $C^{\infty}$-function $f_{P}$ such that $B_{\mid M_{P}}=d A_{P}-* d f_{P}$. Then, $(\widetilde{E}, \widetilde{h}):=(E, h)_{\mid M_{P} \backslash\{P\}}$ with $\widetilde{\nabla}:=\nabla-\sqrt{-1} A_{P}$ id ${ }_{E}$ and $\widetilde{\phi}:=\phi-\sqrt{-1} f_{P} \operatorname{id}_{E}$ is a monopole on $M_{P} \backslash\{\underset{\sim}{P}\}$. If $P$ is Dirac type singularity of $(E, h, \nabla, \phi)$, then $P$ is Dirac type singularity of $(\widetilde{E}, \widetilde{h}, \widetilde{\nabla}, \widetilde{\phi})$. According to [6], it is equivalent to $\left|\widetilde{\phi}_{Q}\right|_{h}=$ $O\left(d(P, Q)^{-1}\right)$ around any point $P \in Z$, which is equivalent to $\left|\phi_{Q}\right|_{h}=O\left(d(P, Q)^{-1}\right)$ around any point $P \in Z$.

\subsection{Twisted difference modules}

Let $\Gamma_{0} \subset \mathbb{C}$ be a lattice. We put $T:=\mathbb{C} / \Gamma_{0}$. Take any $\mathfrak{a} \in T$, and define the automorphism $\Phi$ of $T$ by $\Phi(z)=z+\mathfrak{a}$. Let $\mathfrak{L}$ be a holomorphic line bundle of degree 0 on $T$.

A parabolic $\mathfrak{L}$-twisted difference module $V_{*}=\left(V,\left(\boldsymbol{\tau}_{P}, \mathcal{L}_{P}\right)_{P \in D}\right)$ on $T$ consists of the following data:

- A locally free $\mathcal{O}_{T}$-module $V$ equipped with an isomorphism $V \otimes \mathcal{O}_{T}(* D) \simeq\left(\Phi^{*}\right)^{-1}(V) \otimes$ $\mathfrak{L} \otimes \mathcal{O}_{T}(* D)$, where $D$ is a finite subset of $T$.

- A sequence $0 \leq \tau_{P, 1}<\tau_{P, 2}<\cdots<\tau_{P, m(P)}<1$ for each $P \in D$.

- Lattices $\mathcal{L}_{P, i}(i=1, \ldots, m(P)-1)$ of the stalk $V(* D)_{P}$ at each $P \in D$. We formally set $\mathcal{L}_{P, 0}:=V_{P}$ and $\mathcal{L}_{P, m(P)}:=\left(\left(\Phi^{*}\right)^{-1}(V) \otimes \mathfrak{L}\right)_{P}$ at each $P \in D$. 
The degree of $V_{*}$ is defined by the formula (1.1), i.e.,

$$
\operatorname{deg}\left(V_{*}\right):=\operatorname{deg}(V)+\sum_{P \in D} \sum_{i=1}^{m(P)}\left(1-\tau_{P, i}\right) \operatorname{deg}\left(\mathcal{L}_{P, i}, \mathcal{L}_{P, i-1}\right) .
$$

We set $\mu\left(V_{*}\right):=\operatorname{deg}\left(V_{*}\right) / \operatorname{rank}(V)$.

For any $\mathcal{O}_{T}(* D)$-submodule $0 \neq V^{\prime} \subset V$ such that $V^{\prime}(* D) \simeq\left(\Phi^{*}\right)^{-1}\left(V^{\prime}\right)(* D)$, we obtain lattices $\mathcal{L}_{P, i}^{\prime}$ of $V^{\prime}(* D)_{P}$ by setting $\mathcal{L}_{P, i}^{\prime}:=\mathcal{L}_{P, i} \cap V^{\prime}(* D)_{P}$ in $V(* D)_{P}$, and we obtain a parabolic $\mathfrak{L}$-twisted $\mathfrak{a}$-difference module $V_{*}^{\prime}=\left(V^{\prime},\left(\boldsymbol{\tau}_{P}, \mathcal{L}_{P}^{\prime}\right)_{P \in D}\right)$. Such $V_{*}^{\prime}$ is called a parabolic a-difference submodule of $V_{*}$.

Definition 2.17. $V_{*}$ is called stable (resp. semistable) if

$$
\mu\left(V_{*}^{\prime}\right)<\mu\left(V_{*}\right) \quad\left(\text { resp. } \mu\left(V_{*}^{\prime}\right) \leq \mu\left(V_{*}\right)\right)
$$

for any parabolic a-difference submodules $V^{\prime}$ such that $0<\operatorname{rank} V^{\prime}<\operatorname{rank} V$. It is called polystable if it is semistable and a direct sum of stable objects.

\subsubsection{Example}

It is easy to construct examples of parabolic difference modules.

Lemma 2.18. For any holomorphic line bundle $\mathfrak{L}$ of degree 0 , and for any $d \in \mathbb{R}$, there exists a parabolic $\mathfrak{L}$-difference module $V_{*}$ of rank one such that $\operatorname{deg}\left(V_{*}\right)=d$.

Proof. There exist $P_{1}, \ldots, P_{n} \in T$ and $\ell_{i} \in \mathbb{Z}$ such that $\mathfrak{L}\left(\sum_{i=1}^{n} \ell_{i} P_{i}\right)=\mathcal{O}_{T}$. Note that $\sum \ell_{i}=0$. We take $P_{0} \in T \backslash\left\{P_{1}, \ldots, P_{n}\right\}$. We set $D:=\left\{P_{0}, P_{1}, \ldots, P_{n}\right\}$. We set $V:=\mathcal{O}_{T}$. By our choice of $D$, there exists an isomorphism $F: V(* D) \simeq\left(\Phi^{*}\right)^{-1}(V) \otimes \mathfrak{L}(* D)$. We set $m\left(P_{i}\right)=1$ and $\tau_{P_{i}, 1}=0$ for $i=1, \ldots, n$. We set $m\left(P_{0}\right)=2$, and we choose $0 \leq \tau_{P_{0}, 1}<\tau_{P_{0}, 2}<1$. We set $\mathcal{L}_{P_{0}, 1}=\mathcal{O}_{T}\left(\ell P_{0}\right)_{P_{0}}$ for an integer $\ell$. Then, we obtain a parabolic $\mathfrak{L}$-twisted difference module $V_{*}^{\left(\ell, \tau_{P_{0}, 1}, \tau_{P_{0}, 2}\right)}$ for which $\operatorname{deg}\left(V_{*}^{\left(\ell, \tau_{P_{0}, 1}, \tau_{P_{0}, 2}\right)}\right)=\left(\tau_{P_{0}, 2}-\tau_{P_{0}, 1}\right) \ell$. Then, the claim is clear.

\section{Equivalences}

We shall study equivalences of twisted mini-holomorphic bundles, twisted difference modules, and twisted monopoles. First, in Section 3.1, we introduce analytically stability condition for twisted mini-holomorphic bundles in terms of adapted metrics. We also prepare some formulas for the curvature and the Higgs field of a twisted mini-holomorphic bundle with a Hermitian metric which are standard in the context of mini-holomorphic bundles as in [4]. In Section 3.2, we shall explain the equivalence between twisted mini-holomorphic bundles and twisted difference modules, which preserves the stability conditions. In Section 3.3, we shall explain the equivalence between polystable twisted mini-holomorphic bundles and twisted monopoles.

\subsection{Analytic stability condition for twisted mini-holomorphic bundles}

\subsubsection{3-dimensional torus with mini-complex structure}

We take an oriented base $\left(a_{i}, \alpha_{i}\right)(i=1,2,3)$ of the $\mathbb{R}$-vector space $\mathbb{R} \times \mathbb{C}$. Let $Y:=\mathbb{R} \times \mathbb{C}$ with the Riemannian metric $d t d t+d w d \bar{w}$. It is equipped with the mini-complex structure induced by the mini-complex coordinate system $(t, w)$. We consider the action of $\Gamma:=\mathbb{Z} \mathbf{e}_{1} \oplus \mathbb{Z} \mathbf{e}_{2} \oplus \mathbb{Z} \mathbf{e}_{3}$ on $Y$ given by

$$
\mathrm{e}_{i}(t, w)=(t, w)+\left(a_{i}, \alpha_{i}\right), \quad i=1,2,3 .
$$

Let $\mathcal{M}$ denote the quotient space of $Y$ by the action of $\Gamma$. It is equipped with a naturally induced mini-complex structure. 


\subsubsection{Contraction of the curvature}

Let $Z$ be a finite subset of $\mathcal{M}$. Take $\varrho \in C^{\infty}\left(\mathcal{M}, \Omega^{0,2} \mathcal{M}\right)$. Let $\left(E, \bar{\partial}_{E}\right)$ be a $\varrho$-twisted miniholomorphic bundle on $\mathcal{M} \backslash Z$. Let $h$ be a Hermitian metric of $E$. As in [4], we set

$$
G(h):=\left[\nabla_{h, w}, \nabla_{h, \bar{w}}\right]-\frac{\sqrt{-1}}{2} \nabla_{h, t} \phi_{h} .
$$

If we emphasize the dependence on $\bar{\partial}_{E}$, we use the notation $G\left(h, \bar{\partial}_{E}\right)$. Note that

$$
G(h) d w d \bar{w}=\left(F\left(\nabla_{h}\right)-* \nabla_{h} \phi_{h}\right)^{(1,1)}
$$

for the notation in Section 2.3.2.

Let $U$ be an open subset of $\mathcal{M} \backslash Z$ with $\nu=\nu_{t} d t+\nu_{\bar{w}} d \bar{w} \in C^{\infty}\left(U, \Omega^{0,1}\right)$. On $U$, we set $\bar{\partial}_{E}^{\nu}:=\bar{\partial}_{E}-\nu \operatorname{id}_{E}$. Then, $\left(E_{\mid U}, \bar{\partial}_{E}^{\nu}\right)$ is a $\left(\varrho_{\mid U}-\bar{\partial} \nu\right)$-twisted mini-holomorphic bundle on $U$. We obtain the Chern connection $\nabla_{h}^{\nu}$ and the Higgs field $\phi_{h}^{\nu}$.

Lemma 3.1. The following holds:

$$
\begin{aligned}
& \phi_{h}^{\nu}=\phi_{h}-\sqrt{-1} \operatorname{Re}\left(\nu_{t}\right) \mathrm{id}_{E}, \quad \nabla_{h}^{\nu}=\nabla_{h}-\sqrt{-1}\left(2 \operatorname{Im}\left(\nu_{\bar{w}} d \bar{w}\right)+\operatorname{Im}\left(\nu_{t} d t\right)\right) \operatorname{id}_{E}, \\
& \nabla_{h}^{\nu}\left(\phi_{h}^{\nu}\right)=\nabla_{h}\left(\phi_{h}\right)-\sqrt{-1} d \operatorname{Re}\left(\nu_{t}\right) \operatorname{id}_{E}, \\
& F\left(\nabla_{h}^{\nu}\right)=F\left(\nabla_{h}\right)-\sqrt{-1} d\left(2 \operatorname{Im}\left(\nu_{\bar{w}} d w\right)+\operatorname{Im}\left(\nu_{t}\right) d t\right) \operatorname{id}_{E}, \\
& G\left(h, \bar{\partial}_{E}^{\nu}\right)=G\left(h, \bar{\partial}_{E}\right)-\left(2 \operatorname{Re}\left(\partial_{w} \nu_{\bar{w}}\right)+2^{-1} \operatorname{Re}\left(\partial_{t} \nu_{t}\right)\right) \operatorname{id}_{E} .
\end{aligned}
$$

We can check the formulas by direct computations.

Let $E^{\prime}$ be any $\varrho$-twisted mini-holomorphic subbundle of $E$, i.e., $\bar{\partial}_{E} C^{\infty}\left(\mathcal{M} \backslash Z, E^{\prime}\right) \subset C^{\infty}(\mathcal{M} \backslash$ $\left.Z, \Omega^{0,1} \mathcal{M} \otimes E^{\prime}\right)$. We have the natural $\varrho$-twisted mini-holomorphic structure $\bar{\partial}_{E^{\prime}}$ on $E^{\prime}$. Let $h_{E^{\prime}}$ be the induced metric of $E^{\prime}$. Let $p_{E^{\prime}}$ be the orthogonal projection of $E$ onto $E^{\prime}$ with respect to $h$.

Lemma 3.2. The following Chern-Weil formula holds:

$$
\operatorname{Tr} G\left(h_{E^{\prime}}\right)=\operatorname{Tr}\left(G\left(h_{E}\right) \cdot p_{E^{\prime}}\right)-\left|\partial_{E, \bar{w}} p_{E^{\prime}}\right|^{2}-\frac{1}{4}\left|\partial_{E, t} p_{E^{\prime}}\right|^{2} .
$$

Proof. If $\varrho=0$, it is proved in [4, Section 2.8.2]. Let us study the general case. It is enough to prove the equality locally around any point of $Q \in \mathcal{M} \backslash Z$. On a neighbourhood $U$ of $Q$, there exists $\nu \in C^{\infty}\left(U, \Omega^{0,1}\right)$ such that $\bar{\partial}_{E}^{\nu}$ is a mini-holomorphic structure of $E_{\mid U}$. Note that

$\partial_{E, \bar{w}}^{\nu} p_{E^{\prime}}=\partial_{E, \bar{w}} p_{E^{\prime}}, \partial_{E, t}^{\nu} p_{E^{\prime}}=\partial_{E, t} p_{E^{\prime}}$. Moreover, $\left(E^{\prime}, \bar{\partial}_{E^{\prime}}^{\nu}\right)$ is a mini-holomorphic subbundle of $\left(E, \bar{\partial}_{E}^{\nu}\right)$, and $G\left(h_{E^{\prime}}, \bar{\partial}_{E^{\prime}}^{\nu}\right)=G\left(h_{E^{\prime}}, \bar{\partial}_{E^{\prime}}\right)-\left(2 \operatorname{Re}\left(\partial_{w} \nu \bar{w}\right)+2^{-1} \operatorname{Re}\left(\partial_{t} \nu_{t}\right)\right) \operatorname{id}_{E^{\prime}}$. Then, we obtain the desired formula.

\subsubsection{Analytic stability condition for mini-holomorphic bundles with a Hermitian metric}

Let $\left(E, \bar{\partial}_{E}\right)$ be a $\varrho$-twisted mini-holomorphic bundle on $\mathcal{M} \backslash Z$ with a Hermitian metric $h$.

Definition 3.3. If $\operatorname{Tr} G(h)$ is expressed as a sum of an $L^{1}$-function and a non-positive function, then we set $\operatorname{deg}\left(E, \bar{\partial}_{E}, h\right):=\int_{\mathcal{M} \backslash Z} \operatorname{Tr} G(h) \operatorname{dvol}_{\mathcal{M}} \in \mathbb{R} \cup\{-\infty\}$. We also set $\mu\left(E, \bar{\partial}_{E}, h\right):=$ $\operatorname{deg}\left(E, \bar{\partial}_{E}, h\right) / \operatorname{rank}(E)$.

Suppose that $|G(h)|_{h}$ is $L^{1}$. By $(3.2), \operatorname{deg}\left(E^{\prime}, h_{E^{\prime}}\right)$ is defined in $\mathbb{R} \cup\{-\infty\}$ for any $\varrho$-twisted mini-holomorphic subbundle $E^{\prime}$ of $E$.

Definition 3.4. Suppose that $|G(h)|_{h}$ is $L^{1}$. Then, $\left(E, \bar{\partial}_{E}, h\right)$ is called analytically stable if $\mu\left(E^{\prime}, \bar{\partial}_{E^{\prime}}, h_{E^{\prime}}\right)<\mu\left(E, \bar{\partial}_{E}, h\right)$ for any $\varrho$-twisted mini-holomorphic subbundle $E^{\prime} \subset E$ with $0<\operatorname{rank}\left(E^{\prime}\right)<\operatorname{rank}(E)$. 


\subsubsection{Adapted metrics of twisted mini-holomorphic bundles with Dirac type singularity}

Let $\left(E, \bar{\partial}_{E}\right)$ be a $\varrho$-twisted mini-holomorphic bundle with Dirac type singularity on $(\mathcal{M} ; Z)$.

Lemma 3.5. If $h$ is an adapted metric at $P$, then $G(h)_{Q}=O\left(d(P, Q)^{-1}\right)$ around $P$, where $d(P, Q)$ denotes the distance of $P$ and $Q$. In particular, if $h$ is an adapted metric of $\left(E, \bar{\partial}_{E}\right)$, then $|G(h)|_{h}$ is $L^{1}$.

Proof. In the case $\varrho=0$, it is proved in [4, Lemma 2.35]. The general case follows from Lemma 3.1.

Lemma 3.6. Let $\left(E, \bar{\partial}_{E}\right)$ be a $\varrho$-twisted mini-holomorphic bundle with Dirac type singularity on $(\mathcal{M} ; Z)$. Let $E^{\prime} \neq 0$ be a $\varrho$-twisted mini-holomorphic subbundle of $E$. Let $h$ and $h^{\prime}$ be adapted Hermitian metrics of $E$ and $E^{\prime}$, respectively. Let $h_{E^{\prime}}$ be the metric of $E^{\prime}$ induced by $h$. Then, $\operatorname{deg}\left(E^{\prime}, h_{E^{\prime}}\right)=\operatorname{deg}\left(E^{\prime}, h^{\prime}\right)$.

Proof. It is enough to study the case rank $E^{\prime}=1$. We may assume that there exist neighbourhoods $\mathcal{U}_{P}$ of $P \in Z$ such that $h_{E^{\prime}}=h^{\prime}$ on $\mathcal{M} \backslash \bigcup_{P \in Z} \mathcal{U}_{P}$. Then, we have only to prove that $\int_{\mathcal{U}_{P}} G\left(h^{\prime}\right)=\int_{\mathcal{U}_{P}} G\left(h_{E^{\prime}}\right)$ for any $P \in Z$. By Lemma 3.1, it is enough to study the case $\varrho=0$. It is proved in the proof of [4, Proposition 9.4] (See the argument to compare $\int G\left(h_{0, E_{1}}\right)$ and $\int G\left(h_{2, E_{1}}\right)$ in the proof of [4, Proposition 9.4].)

Corollary 3.7. If $h_{1}$ and $h_{2}$ are adapted metrics of $\left(E, \bar{\partial}_{E}\right), \operatorname{deg}\left(E, \bar{\partial}_{E}, h_{1}\right)=\operatorname{deg}\left(E, \bar{\partial}_{E}, h_{2}\right)$ holds.

Lemma 3.8. Take a small neighbourhood $\mathcal{U}_{P}$ of $P \in Z$. The following estimates hold for $Q \in \mathcal{U}_{P} \backslash\{P\}:$

$$
\begin{aligned}
& \left|\phi_{h, Q}\right|_{h}=O\left(d(P, Q)^{-1}\right), \quad\left|\left(\nabla \phi_{h}\right)_{Q}\right|_{h, g_{\mathcal{M}}}=O\left(d(P, Q)^{-2}\right), \\
& \left|F\left(\nabla_{h}\right)_{Q}\right|_{h, g_{\mathcal{M}}}=O\left(d(P, Q)^{-2}\right) .
\end{aligned}
$$

In particular, $\left|\nabla_{h} \phi_{h}\right|_{h}$ and $\left|F\left(\nabla_{h}\right)\right|_{h}$ are $L^{1}$.

Proof. Suppose that $\varrho=0$. The estimates $\left|\phi_{h, Q}\right|_{h}=O\left(d(P, Q)^{-1}\right)$ and $\left|\left(\nabla \phi_{h}\right)_{Q}\right|_{h, g_{\mathcal{M}}}=$ $O\left(d(P, Q)^{-2}\right)$ directly follow from [6, Proposition 1]. Because of Lemma 2.12, Lemma 3.5 and (3.1), we obtain $\left|F\left(\nabla_{h}\right)_{Q}\right|_{h, g_{\mathcal{M}}}=O\left(d(P, Q)^{-2}\right)$. We can reduce the case $\varrho$ to the case $\varrho=0$ by using Lemma 3.1 .

\subsubsection{Analytic stability condition for $\varrho$-twisted mini-holomorphic bundles with Dirac type singularity}

Let $\left(E, \bar{\partial}_{E}\right)$ be a $\varrho$-twisted mini-holomorphic bundle with Dirac type singularity on $(\mathcal{M} ; Z)$. We set

$$
\operatorname{deg}^{\text {an }}\left(E, \bar{\partial}_{E}\right):=\operatorname{deg}\left(E, \bar{\partial}_{E}, h\right), \quad \mu^{\text {an }}\left(E, \bar{\partial}_{E}\right):=\operatorname{deg}^{\text {an }}\left(E, \bar{\partial}_{E}\right) / \operatorname{rank}(E)
$$

for an adapted Hermitian metric $h$ of $E$, which is independent of the choice of $h$. The numbers are called the analytic degree and the analytic slope of $\left(E, \bar{\partial}_{E}, h\right)$, respectively.

Definition 3.9. We say that $\left(E, \bar{\partial}_{E}\right)$ is analytically stable if $\mu^{\text {an }}\left(E^{\prime}, \bar{\partial}_{E^{\prime}}\right)<\mu^{\text {an }}\left(E, \bar{\partial}_{E}\right)$ holds for any $\varrho$-twisted mini-holomorphic subbundle $E^{\prime} \subset E$ with $0<\operatorname{rank}\left(E^{\prime}\right)<\operatorname{rank}(E)$. It is called polystable if $\left(E, \bar{\partial}_{E}\right)=\bigoplus\left(E_{i}, \bar{\partial}_{E_{i}}\right)$, where each $\left(E_{i}, \bar{\partial}_{E_{i}}\right)$ is stable such that $\mu^{\text {an }}\left(E_{i}, \bar{\partial}_{E_{i}}\right)=$ $\mu^{\text {an }}\left(E, \bar{\partial}_{E}\right)$. 
We obtain the following lemma from Lemma 3.6.

Lemma 3.10. A @-twisted mini-holomorphic bundle with Dirac type singularity $\left(E, \bar{\partial}_{E}\right)$ on $(\mathcal{M} ; Z)$ is analytically stable if and only if $\left(E, \bar{\partial}_{E}, h\right)$ is analytically stable for an adapted Hermitian metric $h$ of $E$.

\subsubsection{Complement on the choice of $\varrho$}

Let $H^{i}\left(\mathcal{M}, \mathcal{O}_{\mathcal{M}}\right)$ denote the $i$-th cohomology group of the complex $\left(C^{\infty}\left(\mathcal{M}, \Omega^{0, i} \mathcal{M}\right), \bar{\partial}_{\mathcal{M}}\right)$. For any $\nu \in C^{\infty}\left(\mathcal{M}, \Omega^{0,1} \mathcal{M}\right), \varrho$-twisted mini-holomorphic bundles are equivalent to $(\varrho-\bar{\partial} \nu)$-twisted mini-holomorphic bundles. Hence, the essential ambiguity of the choice of $\varrho$ lives in $H^{2}\left(\mathcal{M}, \mathcal{O}_{\mathcal{M}}\right)$.

Lemma 3.11. We have the following isomorphisms:

$$
H^{0}\left(\mathcal{M}, \mathcal{O}_{\mathcal{M}}\right) \simeq \mathbb{C}, \quad H^{1}\left(\mathcal{M}, \mathcal{O}_{\mathcal{M}}\right) \simeq \mathbb{C} d t \oplus \mathbb{C} d \bar{w}, \quad H^{2}\left(\mathcal{M}, \mathcal{O}_{\mathcal{M}}\right) \simeq \mathbb{C} d t \wedge d \bar{w}
$$

Hence, for the study of twisted mini-holomorphic bundles, it is essential to study the case $\varrho=$ $\alpha d t d \bar{w}$ for some $\alpha \in \mathbb{C}$.

Proof. We have the isomorphism $\mathbb{R}_{s} \times\left(\mathbb{R}_{t} \times \mathbb{C}_{w}\right) \simeq \mathbb{C}_{z} \times \mathbb{C}_{w}$ given by $(s, t, w) \longmapsto(s+\sqrt{-1} t, w)$. We consider the action of $\mathbb{Z} \times \Gamma$ on $\mathbb{R} \times(\mathbb{R} \times \mathbb{C})$ induced by the natural action of $\mathbb{Z}$ on $\mathbb{R}$ and the $\Gamma$-action on $\mathbb{R} \times \mathbb{C}$. Let $X$ denote the quotient space. We have the projection $\varphi: X \longrightarrow \mathcal{M}$ induced by $(s, t, w) \longmapsto(t, w)$. We have the natural $S^{1}=\mathbb{R} / \mathbb{Z}$-action on $X$, and the quotient space is identified with $\mathcal{M}$. Let $\varphi^{*}: C^{\infty}\left(\mathcal{M}, \Omega^{0, i} \mathcal{M}\right) \longrightarrow C^{\infty}\left(X, \Omega^{0, i}(X)\right)$ be the map induced by $\varphi^{*}(d \bar{w})=d \bar{w}, \varphi^{*}(d t)=\partial_{\bar{z}}(t) d \bar{z}=\frac{\sqrt{-1}}{2} d \bar{z}$ and the natural pull back $\varphi^{*}: C^{\infty}(\mathcal{M}, \mathbb{C}) \longrightarrow$ $C^{\infty}(X, \mathbb{C})$. Then, it is easy to check that it is a morphism of complexes, and that it induces an isomorphism between $C^{\infty}\left(\mathcal{M}, \Omega^{0, \bullet} \mathcal{M}\right)$ and the $S^{1}$-invariant part of $C^{\infty}\left(X, \Omega^{0, \bullet}(X)\right)$. Therefore, it induces the isomorphism of $H^{i}\left(\mathcal{M}, \mathcal{O}_{\mathcal{M}}\right)$ and the $S^{1}$-invariant part of $H^{i}\left(X, \mathcal{O}_{X}\right)$. Then, the claim of the lemma follows.

Remark 3.12. Let $\mathcal{M}=\bigcup_{\lambda \in \Lambda} U_{\lambda}$ be an open covering such that the following holds:

- There exist $\nu_{\lambda} \in C^{\infty}\left(U_{\lambda}, \Omega_{U_{\lambda}}^{0,1}\right)$ such that $\varrho_{\mid U_{\lambda}}=\bar{\partial} \nu_{\lambda}$.

- There exist $\alpha_{\lambda, \mu} \in C^{\infty}\left(U_{\lambda} \cap U_{\mu}\right)$ such that $\nu_{\lambda}-\nu_{\mu}=\bar{\partial} \alpha_{\lambda, \mu}$. We assume that $\alpha_{\lambda, \lambda}=0$ and $\alpha_{\lambda, \mu}=-\alpha_{\mu, \lambda}$.

Let $\mathcal{E}_{\lambda}$ be the $\mathcal{O}_{U_{\lambda}}$-module obtained as the sheaf of mini-holomorphic sections of $\left(E_{U_{\lambda}}, \bar{\partial}_{E}-\nu_{\lambda}\right)$. We obtain the isomorphism $\beta_{\lambda, \mu}: \mathcal{E}_{\lambda \mid U_{\lambda} \cap U_{\mu}} \simeq \mathcal{E}_{\mu \mid U_{\lambda} \cap U_{\mu}}$ by the multiplication of $\exp \left(-\alpha_{\lambda, \mu}\right)$. We obtain the holomorphic functions $\theta_{\lambda, \mu, \kappa}$ on $U_{\lambda, \mu, \kappa}$ such that $\beta_{\lambda, \mu} \circ \beta_{\mu, \kappa} \circ \beta_{\kappa, \lambda}=\theta_{\lambda, \mu, \kappa}$ id. Such a tuple $\left(\left\{\mathcal{E}_{\lambda}\right\},\left\{\beta_{\lambda, \mu}\right\}\right)$ is called a twisted sheaf. The cohomology class of $\left[\theta_{\lambda, \mu, \kappa}\right]$ in $H^{2}\left(\mathcal{M}, \mathcal{O}_{\mathcal{M}}^{*}\right)$ depends only on $\varrho$, and it is equal to the image of $\varrho$ via the natural map $H^{2}\left(\mathcal{M}, \mathcal{O}_{\mathcal{M}}\right) \longrightarrow$ $H^{2}\left(\mathcal{M}, \mathcal{O}_{\mathcal{M}}^{*}\right)$.

\subsection{Twisted difference modules and twisted mini-holomorphic bundles}

We assume that (i) the tuple $\left(a_{i}, \alpha_{i}\right)(i=1,2,3)$ is an oriented base of $\mathbb{R} \times \mathbb{C}$, (ii) $\alpha_{1}$ and $\alpha_{2}$ are linearly independent over $\mathbb{R}$, (iii) the tuple $\left(\alpha_{1}, \alpha_{2}\right)$ is an oriented base of $\mathbb{C}$. Let $\Gamma_{0} \subset \mathbb{C}$ be the lattice generated by $\alpha_{1}$ and $\alpha_{2}$.

Let $\mathcal{M}^{\text {cov }}$ denote the quotient space of $Y$ by the action of $\mathbb{Z} \mathbf{e}_{1} \oplus \mathbb{Z} \mathbf{e}_{2}$. We have the natural isomorphism $\mathcal{M}^{\text {cov }} / \mathbb{Z}_{3} \simeq \mathcal{M}$. The projection $Y \longrightarrow \mathbb{C}$ induces a morphism $\mathcal{M}^{\text {cov }} \longrightarrow T:=$ $\mathbb{C} / \Gamma_{0}$. 


\subsubsection{Another mini-complex coordinate system}

We introduce another mini-complex coordinate system $(s, u)$ on $Y$. We set

$$
\gamma:=-\frac{a_{1} \bar{\alpha}_{2}-a_{2} \bar{\alpha}_{1}}{\alpha_{1} \bar{\alpha}_{2}-\alpha_{2} \bar{\alpha}_{1}}
$$

We introduce another mini-complex coordinate system $(s, u)$ on the mini-complex manifold $Y$ as follows:

$$
s:=t+2 \operatorname{Re}(\gamma w)=t+\overline{\gamma w}+\gamma w, \quad u:=w .
$$

Then, we obtain $\mathrm{e}_{i}(s, u)=\left(s, u+\alpha_{i}\right)$ for $i=1,2$. We also obtain $\mathrm{e}_{3}(s, u)=(s+\mathfrak{t}, u+\mathfrak{a})$, where

$$
\mathfrak{t}:=a_{3}+2 \operatorname{Re}\left(\gamma \alpha_{3}\right), \quad \mathfrak{a}:=\alpha_{3} .
$$

Note that $\mathfrak{t}>0$, which follows from that the tuple $\left\{\left(a_{i}, \alpha_{i}\right)\right\}_{i=1,2,3}$ is an oriented base of $\mathbb{R} \times \mathbb{C}$, and that $\left\{\alpha_{1}, \alpha_{2}\right\}$ is an oriented base of $\mathbb{C}$. We have the following relations of complex vector fields:

$$
\partial_{\bar{w}}=\partial_{\bar{u}}+\bar{\gamma} \partial_{s}, \quad \partial_{w}=\partial_{u}+\gamma \partial_{s}, \quad \partial_{t}=\partial_{s} .
$$

The product $\mathbb{R}_{s} \times T$ is equipped with the natural mini-complex structure. The mini-complex coordinate system $(s, u)$ induces an isomorphism of mini-complex manifolds $\mathcal{M}^{\text {cov }} \simeq \mathbb{R}_{s} \times T$.

\subsubsection{Twisted mini-holomorphic bundles and twisted difference modules}

Let $Z$ be a finite subset in $\mathcal{M}$. Let $Z^{\text {cov }} \subset \mathcal{M}^{\text {cov }} \simeq \mathbb{R}_{s} \times T$ denote the pull back of $Z$. For any $a<b$, we set $\left[a, b\left[:=\{a \leq s<b\}\right.\right.$. We take $\epsilon>0$ such that $\left(\left[-\epsilon, 0[\times T) \cap Z^{\text {cov }}=\varnothing\right.\right.$. Let $D$ be the image of $Z^{\text {cov }} \cap\left(\left[-\epsilon, \mathfrak{t}[\times T)\right.\right.$ via the projection $\mathbb{R}_{s} \times T \longrightarrow T$. For each $P \in D$, we obtain the sequence $0 \leq s_{P, 1}<s_{P, 2}<\cdots<s_{P, m(P)}<\mathfrak{t}$ by the condition:

$$
\left\{\left(s_{P, i}, P\right) \mid i=1, \ldots, m(P)\right\}=\left(\left[0, \mathfrak{t}[\times\{P\}) \cap Z^{\mathrm{cov}} .\right.\right.
$$

We set $\tau_{P, i}:=s_{P, i} / \mathfrak{t}$.

We have the expression $\varrho=\varrho_{0} d t d \bar{w}=\varrho_{0} d s d \bar{u}$. Let $\varrho_{0}^{\text {cov }}$ be the function on $\mathcal{M}^{\text {cov }}=\mathbb{R}_{s} \times T$ obtained as the pull back of $\varrho_{0}$ by $\mathcal{M}^{\text {cov }} \longrightarrow \mathcal{M}$. We define $\nu_{\varrho}=\nu_{\varrho}, \bar{w} d \bar{w} \in C^{\infty}\left(\mathcal{M}^{\text {cov }}, \Omega^{0,1} \mathcal{M}\right)$ by setting

$$
\nu_{\varrho, \bar{w}}(s, u)=\int_{0}^{s} \varrho_{0}^{\operatorname{cov}}(\sigma, u) d \sigma .
$$

We set $\vartheta_{\varrho}:=\nu_{\varrho \mid\{t\} \times T}$. Let $\mathfrak{L}_{\varrho}$ be the holomorphic line bundle on $T$ given by the product bundle $\mathbb{C} \times T$ with $\bar{\partial}_{T}-\vartheta_{\varrho}$.

Let $\left(E, \bar{\partial}_{E}\right)$ be a $\varrho$-twisted mini-holomorphic bundle with Dirac type singularity on $(\mathcal{M} ; Z)$. Let us observe that $\left(E, \bar{\partial}_{E}\right)$ induces a parabolic $\mathfrak{L}_{\varrho^{-}}$twisted difference module $\Upsilon\left(E, \bar{\partial}_{E}\right)$ over $\left(T,\left(\boldsymbol{\tau}_{P}\right)_{P \in D}\right)$.

Let $\varrho^{\text {cov }} \in C^{\infty}\left(\mathcal{M}, \Omega^{0,1} \mathcal{M}\right)$ be the pull back of $\varrho$. Let $\left(E^{\text {cov }}, \bar{\partial}_{E^{\text {cov }}}\right)$ denote the $\varrho^{\text {cov }}$-twisted mini-holomorphic bundle on $\mathcal{M}^{\text {cov }}$ obtained as the pull back of $\left(E, \bar{\partial}_{E}\right)$. We set $\left(\widetilde{E}^{\text {cov }}, \bar{\partial}_{\widetilde{E}^{\text {cov }}}\right):=$ $\left(E^{\text {cov }}, \bar{\partial}_{E^{\text {cov }}}-\nu_{\varrho}\right)$ which is a mini-holomorphic bundle on $\mathcal{M}^{\text {cov }}$.

Let $V$ be the locally free $\mathcal{O}_{T}$-module obtained as $\widetilde{E}_{\mid\{-\epsilon\} \times T}^{\text {cov }}$. It is independent of the choice of $\epsilon$ as above, up to canonical isomorphisms.

Let $\Phi: T \longrightarrow T$ be the morphism induced by $\Phi(u)=u+\mathfrak{a}$. We have the natural isomorphism

$$
\Phi^{*}\left(E_{\mid\{\mathfrak{t}-\epsilon\} \times T}^{\mathrm{cov}}\right) \simeq E_{\mid\{-\epsilon\} \times T}^{\mathrm{cov}} .
$$


It induces the following isomorphism of holomorphic bundles on $T$ :

$$
\Phi^{*}\left(\left(\widetilde{E}^{\text {cov }}, \bar{\partial}_{\widetilde{E}^{\text {cov }}}\right)_{\mid\{\mathfrak{t}-\epsilon\} \times T}\right) \simeq\left(\widetilde{E}^{\text {cov }}, \bar{\partial}_{\widetilde{E}^{\text {cov }}}\right)_{\mid\{-\epsilon\} \times T} \otimes \mathfrak{L}_{\varrho} .
$$

The scattering map induces an isomorphism

$$
\left(\widetilde{E}^{\mathrm{cov}}, \bar{\partial}_{\widetilde{E}^{\mathrm{cov}}}\right)_{\mid\{-\epsilon\} \times T}(* D) \simeq\left(\widetilde{E}^{\mathrm{cov}}, \bar{\partial}_{\widetilde{E}^{\mathrm{cov}}}\right)_{\mid\{\mathfrak{t}-\epsilon\} \times T}(* D) .
$$

Hence, $V$ is equipped with an isomorphism $V(* D) \simeq\left(\left(\Phi^{*}\right)^{-1}(V) \otimes \mathfrak{L}_{\varrho}\right)(* D)$.

For each $P \in D$ and for $i=1, \ldots, m(P)-1$, we take $s_{P, i}<b_{P, i}<s_{P, i+1}$. Let $\left(\widetilde{E}_{\mid\{-\epsilon\} \times T}^{\text {cov }}\right)_{P}$ denote the $\mathcal{O}_{T, P}$-module obtained as the stalk of the sheaf of holomorphic sections of $\widetilde{E}_{\mid\{-\epsilon\} \times T}^{\text {cov }}$ at $P$. Similarly, $\left(\widetilde{E}_{\mid\left\{b_{P, i}\right\} \times T}^{\mathrm{cov}}\right)_{P}$ denote the $\mathcal{O}_{T, P^{-}}$-module obtained as the stalk of the sheaf of holomorphic sections of $\widetilde{E}_{\mid\left\{b_{P, i}\right\} \times T}^{\text {cov }}$ at $P$. The scattering map induces isomorphisms of $\mathcal{O}_{T}(* P)_{P^{-}}$ modules:

$$
\left(\widetilde{E}_{\mid\{-\epsilon\} \times T}^{\text {cov }}\right)_{P}(* P) \simeq\left(\widetilde{E}_{\mid\left\{b_{P, i}\right\} \times T}^{\text {cov }}\right)_{P}(* P) .
$$

Hence, $\left(E_{\mid\left\{b_{P, i}\right\} \times T}^{\text {cov }}\right)_{P}(i=1, \ldots, m(P)-1)$ induce a sequence of lattices $\mathcal{L}_{P, i}(i=1, \ldots, m(P)-1)$ of $V(* D)_{P}$. Thus, we obtain the following parabolic a-difference module on $\left(T,\left(\boldsymbol{\tau}_{P}\right)_{P \in D}\right)$ :

$$
\Upsilon\left(E, \bar{\partial}_{E}\right):=\left(V,\left(\boldsymbol{\tau}_{P}, \mathcal{L}_{P}\right)_{P \in D}\right) .
$$

The following proposition is clear by the construction.

Proposition 3.13. $\Upsilon$ induces an equivalence between $\varrho$-twisted mini-holomorphic bundles with Dirac type singularity on $(\mathcal{M} ; Z)$ and parabolic $\mathfrak{L}_{\varrho}$-twisted $\mathfrak{a}$-difference modules on $\left(T,\left(\boldsymbol{\tau}_{P}\right)_{P \in D}\right)$.

\subsubsection{Comparison of stability conditions}

Let $\left(E, \bar{\partial}_{E}\right)$ be a $\varrho$-mini-holomorphic bundle with Dirac type singularity on $(\mathcal{M} ; Z)$.

Proposition 3.14. We have $\mu^{\text {an }}\left(E, \bar{\partial}_{E}\right)=\mathfrak{t} \pi \mu\left(\Upsilon\left(E, \bar{\partial}_{E}\right)\right)+2 \int_{\mathcal{M}} \operatorname{Re}\left(\gamma \varrho_{0}\right)$. As a result, $\left(E, \bar{\partial}_{E}\right)$ is analytically (poly)stable if and only if $\Upsilon\left(E, \bar{\partial}_{E}\right)$ is (poly)stable.

Proof. We consider the real vector field $\mathfrak{v}:=2 \bar{\gamma} \partial_{w}+2 \gamma \partial_{\bar{w}}-\left(2|\gamma|^{2}-\frac{1}{2}\right) \partial_{t}$ on $\mathcal{M}$. Let $h$ be any Hermitian metric of $E$. Let $\partial_{E, \bar{u}}$ denote the operator on $E$ induced by $\bar{\partial}_{E}$ and $\partial_{\bar{u}}$. Let $\partial_{E, h, u}$ denote the operator on $E$ induced by $\partial_{E, h}$ and $\partial_{u}$.

Lemma 3.15. $G(h)=\left[\partial_{E, h, u}, \partial_{E, \bar{u}}\right]-\sqrt{-1} \nabla_{h, \mathfrak{v}} \phi_{h}+2 \operatorname{Re}\left(\varrho_{0}\right) \operatorname{id}_{E}$ holds.

Proof. Because $\partial_{E, t}=\nabla_{h, t}-\sqrt{-1} \phi_{h}$ and $\partial_{E, h, t}=\nabla_{h, t}+\sqrt{-1} \phi_{h}$, the following holds:

$$
\partial_{E, \bar{u}}=\nabla_{h, \bar{w}}-\bar{\gamma}\left(\nabla_{h, t}-\sqrt{-1} \phi_{h}\right), \quad \partial_{E, h, u}=\nabla_{h, w}-\gamma\left(\nabla_{h, t}+\sqrt{-1} \phi_{h}\right) .
$$

Hence, we obtain

$$
\begin{aligned}
{\left[\partial_{E, h, u}, \partial_{E, \bar{u}}\right]=} & {\left[\nabla_{h, w}, \nabla_{h, \bar{w}}\right]-\bar{\gamma}\left[\nabla_{h, w}, \nabla_{h, t}\right]+\bar{\gamma} \sqrt{-1} \nabla_{h, w} \phi_{h} } \\
& +\gamma\left[\nabla_{h, \bar{w}}, \nabla_{h, t}\right]+\gamma \sqrt{-1} \nabla_{h, \bar{w}} \phi-2 \sqrt{-1}|\gamma|^{2} \nabla_{h, t} \phi_{h} .
\end{aligned}
$$

According to Lemma 2.12 , we have $\left[\nabla_{h, \bar{w}}, \nabla_{h, t}\right]-\sqrt{-1} \nabla_{h, \bar{w}} \phi=-\varrho_{0} \mathrm{id}_{E}$ and $\left[\nabla_{h, w}, \nabla_{h, t}\right]+$ $\sqrt{-1} \nabla_{h, w} \phi=\bar{\varrho}_{0} \mathrm{id}_{E}$. Hence, we obtain

$$
\begin{aligned}
{\left[\partial_{E, h, u}, \partial_{E, \bar{u}}\right]=} & {\left[\nabla_{h, w}, \nabla_{h, \bar{w}}\right]+2 \sqrt{-1} \bar{\gamma} \nabla_{w} \phi+2 \sqrt{-1} \gamma \nabla_{\bar{w}} \phi } \\
& -2 \sqrt{-1}|\gamma|^{2} \nabla_{t} \phi-2 \operatorname{Re}\left(\gamma \varrho_{0}\right) \operatorname{id}_{E} .
\end{aligned}
$$

Then, we obtain the claim of the lemma. 
Let $h$ be an adapted metric of $\left(E, \bar{\partial}_{E}\right)$. According to Lemmas 3.5 and $3.8, G(h)$ and $\nabla_{h} \phi_{h}$ are $L^{1}$. Hence, we obtain

$$
\begin{aligned}
\operatorname{deg}^{\mathrm{an}}(E)= & \int_{\mathcal{M}} \operatorname{Tr} G(h)=\int_{\mathcal{M}} \operatorname{Tr}\left[\partial_{E, h, u}, \partial_{E, \bar{u}}\right] \\
& -\int_{\mathcal{M}} \sqrt{-1} \operatorname{Tr} \nabla_{h, \mathfrak{v}} \phi_{h}+2 \operatorname{rank}(E) \int_{\mathcal{M}} \operatorname{Re}\left(\gamma \varrho_{0}\right) .
\end{aligned}
$$

Note that the volume form of $\mathcal{M}$ is equal to $\frac{\sqrt{-1}}{2} d t d w d \bar{w}=\frac{\sqrt{-1}}{2} d s d u d \bar{u}$. By the Stokes theorem and the estimate in Lemma 3.8, we obtain that $\int_{\mathcal{M}} \operatorname{Tr}\left(\nabla_{h, \mathfrak{v}} \phi_{h}\right) \frac{\sqrt{-1}}{2} d t d w d \bar{w}=0$. By the Fubini theorem, we obtain that

$$
\begin{aligned}
\int_{\mathcal{M}} \operatorname{Tr}\left[\partial_{E, h, u}, \partial_{E, \bar{u}}\right] & =\int_{0}^{\mathfrak{t}} d s \int_{\{s\} \times T} \operatorname{Tr}\left[\partial_{E, h, u}, \partial_{E, \bar{u}}\right] \frac{\sqrt{-1}}{2} d u d \bar{u} \\
& =\int_{0}^{\mathfrak{t}} d s \int_{\{s\} \times T} \pi c_{1}\left(E_{\mid\{s\} \times T}^{\mathrm{cov}}\right)=\mathfrak{t} \pi \operatorname{deg} \Upsilon\left(E, \bar{\partial}_{E}\right) .
\end{aligned}
$$

Thus, we obtain the claim of Proposition 3.14.

\subsection{Twisted monopoles and twisted mini-holomorphic bundles}

\subsubsection{Statements}

Let $B$ be a real 2 -form on $\mathcal{M}$. We set $\varrho_{B}:=\sqrt{-1} B^{(0,1), \eta}$ and $\mu_{B}:=-\frac{1}{2} \int_{\mathcal{M}} B^{(1,1)} d t$. Let $(E, h, \nabla, \phi)$ be a $B$-twisted monopole with Dirac type singularity on $\mathcal{M} \backslash Z$. We have the associated $\varrho_{B}$-twisted mini-holomorphic bundle $\left(E, \bar{\partial}_{E}\right)$. Note that $G\left(h, \bar{\partial}_{E}\right) d w d \bar{w}=\sqrt{-1} B^{(1,1)} \mathrm{id}_{E}$. Hence, we obtain

$$
\mu^{\mathrm{an}}\left(E, \bar{\partial}_{E}\right)=\frac{1}{\operatorname{rank}(E)} \int_{\mathcal{M}} \operatorname{Tr}\left(G\left(h, \bar{\partial}_{E}\right)\right) \frac{\sqrt{-1}}{2} d t d w d \bar{w}=-\frac{1}{2} \int_{\mathcal{M}} B^{(1,1)} d t=\mu_{B}
$$

We shall prove the following theorem in Sections 3.3.2-3.3.4, which is a variant of the correspondence in [1] on the basis of [7].

Theorem 3.16. The above construction induces an equivalence between B-twisted monopoles with Dirac type singularity on $\mathcal{M} \backslash Z$ and analytically polystable $\varrho_{B}$-twisted mini-holomorphic bundles with Dirac type singularity with slope $\mu_{B}$ on $(\mathcal{M} ; Z)$.

More precisely, Theorem 3.16 consists of Propositions 3.18, 3.19, and 3.21 below.

Remark 3.17. According to Lemma 2.9 and Remark 2.10, it is essential to study the case where

$$
B=c \frac{\sqrt{-1}}{2} d w \wedge d \bar{w}+\alpha d t \wedge d \bar{w}+\bar{\alpha} d t \wedge d w
$$

for $(c, \alpha) \in \mathbb{R} \times \mathbb{C}$. We have $\varrho_{B}=\sqrt{-1} \alpha d t \wedge d w$ and $\mu_{B}=-\frac{1}{2} \operatorname{vol}(\mathcal{M}) c$ in this case.

\subsubsection{Polystability}

Let $\left(E, \bar{\partial}_{E}, h\right)$ be a $B$-twisted monopole with Dirac type singularity on $\mathcal{M} \backslash Z$.

Proposition 3.18. $\left(E, \bar{\partial}_{E}\right)$ is analytically polystable with $\operatorname{deg}^{\text {an }}\left(E, \bar{\partial}_{E}\right)=\operatorname{rank}(E) \mu_{B}$. 
Proof. Let $E^{\prime}$ be a $\varrho_{B}$-twisted mini-holomorphic subbundle of $E$. Let $h_{E^{\prime}}$ be the metric of $E^{\prime}$ induced by $h$. By the Chern-Weil formula (3.2) and Lemma 3.6, we have

$$
\begin{aligned}
\operatorname{deg}^{\text {an }}\left(E^{\prime}, \bar{\partial}_{E^{\prime}}\right)= & \int \operatorname{Tr} G\left(h_{E^{\prime}}\right)=\operatorname{rank}\left(E^{\prime}\right) \mu_{B}-\int\left|\partial_{E, \bar{w}} p_{E^{\prime}}\right|^{2} \\
& -\frac{1}{4} \int\left|\partial_{E, t} p_{E^{\prime}}\right|^{2} \leq \operatorname{rank}\left(E^{\prime}\right) \mu_{B} .
\end{aligned}
$$

If $\mu^{\text {an }}\left(E^{\prime}, \bar{\partial}_{E^{\prime}}\right)=\mu_{B}$, we obtain $\partial_{E, \bar{w}} p_{E^{\prime}}=\partial_{E, t} p_{E^{\prime}}=0$. We obtain that the orthogonal complement $E^{\prime \perp}$ is also a $\varrho_{B}$-twisted mini-holomorphic subbundle of $E$. Let $h_{E^{\prime \perp}}$ be the metric of $E^{\prime \perp}$ induced by $h$. Thus, we obtain a decomposition of monopoles $\left(E, \bar{\partial}_{E}, h\right)=$ $\left(E^{\prime}, \bar{\partial}_{E^{\prime}}, h_{E^{\prime}}\right) \oplus\left(E^{\prime \perp}, \bar{\partial}_{E^{\prime \perp}}, h_{E^{\prime}}\right)$. Hence, we obtain the polystability of $\left(E, \bar{\partial}_{E}\right)$ by an easy induction.

\subsubsection{Uniqueness}

The uniqueness is also standard.

Proposition 3.19. Let $\left(E, \bar{\partial}_{E}\right)$ be a $\varrho_{B}$-twisted mini-holomorphic bundle with Dirac type singularity on $(\mathcal{M} ; Z)$. Let $h_{1}$ and $h_{2}$ be adapted Hermitian-metrics of $E$ such that $G\left(h_{i}\right) d w d \bar{w}=$ $\sqrt{-1} B^{(1,1)} \mathrm{id}_{E}$. Then, there exists a decomposition $\left(E, \bar{\partial}_{E}\right)=\bigoplus\left(E_{j}, \bar{\partial}_{E_{j}}\right)$ such that $(i)$ it is orthogonal with respect to both $h_{1}$ and $h_{2}$, (ii) there exist positive constants $a_{j}$ such that $h_{2 \mid E_{j}}=a_{j} h_{1 \mid E_{j}}$.

Proof. Let $s$ be the automorphism of $E$ determined by $h_{2}=h_{1} s$.

Lemma 3.20. The following inequality holds on $\mathcal{M} \backslash Z$ :

$$
-\left(\partial_{\bar{w}} \partial_{w}+\frac{1}{4} \partial_{t}^{2}\right) \operatorname{Tr}(s)=-\left|s^{-1 / 2} \partial_{E, h_{1}, w}(s)\right|_{h_{1}}^{2}-\frac{1}{4}\left|s^{-1 / 2} \partial_{E, h_{1}, t}(s)\right|_{h_{1}}^{2} \leq 0
$$

Proof. In the case $\varrho_{B}=0$, it follows from [4, Corollary 2.30]. (Note that $\partial_{E, h_{1}, t}$ is denoted by $\partial_{E, h_{1}, t}^{\prime}$ in [4, Corollary 2.30].) Let us study the general case. We have only to check the inequality locally around any point $P$ of $\mathcal{M} \backslash Z$. We take a small neighbourhood $U$ of $P$ and $\nu=\nu_{t} d t+\nu_{\bar{w}} d \bar{w} \in C^{\infty}\left(U, \Omega^{0,1}\right)$ such that $\bar{\partial}_{E}-\nu$ id is mini-holomorphic. We obtain $\partial_{E, h, w}^{\nu}=\partial_{E, h, w}+\overline{\nu_{\bar{w}}}$ id and $\partial_{E, h, t}^{\nu}=\partial_{E, h, t}+\overline{\nu_{t}}$ id. Hence, we obtain $\partial_{E, h, w}^{\nu}(s)=\left[\partial_{E, h, w}^{\nu}, s\right]=$ $\left[\partial_{E, h, w}, s\right]=\partial_{E, h, w}(s)$. Similarly, we obtain $\partial_{E, h, t}^{\nu}(s)=\partial_{E, h, t}(s)$. Hence, the general case can be reduced to the case $\varrho_{B}=0$.

By the assumption, $\operatorname{Tr}(s) \geq 0$ is bounded. Then, the inequality holds on $\mathcal{M}$ in the sense of distributions. (See the proof of [7, Proposition 2.2].) Hence, we obtain that $\operatorname{Tr}(s)$ is constant, and $\partial_{E, h_{1}, w}(s)=\partial_{E, h_{1}, t}(s)=0$. Because $s$ is self-adjoint with respect to $h_{1}$, we also obtain that $\partial_{E, \bar{w}}(s)=\partial_{E, t}(s)=0$. We obtain that the eigenvalues of $s$ are constant, and the eigen decomposition $E=\bigoplus E_{i}$ is compatible with the mini-holomorphic structure. Then, the claim of the proposition follows.

\subsubsection{Construction of twisted monopoles}

Let $\left(E, \bar{\partial}_{E}\right)$ be a stable $\varrho_{B}$-twisted mini-holomorphic bundle with Dirac type singularity on $(\mathcal{M} ; Z)$ with $\mu^{\text {an }}\left(E, \bar{\partial}_{E}\right)=\mu_{B}$.

Proposition 3.21. There exists a Hermitian metric $h$ of $\left(E, \bar{\partial}_{E}\right)$ such that $\left(E, \bar{\partial}_{E}, h\right)$ is a Btwisted monopole with Dirac type singularity on $\mathcal{M} \backslash Z$. 
Proof. As a preliminary, let us consider the rank one case. Note that the stability condition is trivial in the rank one case.

Lemma 3.22. Assume $\operatorname{rank} E=1$. Then, there exists a Hermitian metric $h$ of $\left(E, \bar{\partial}_{E}\right)$ such that $\left(E, \bar{\partial}_{E}, h\right)$ is a B-twisted monopole with Dirac type singularity on $\mathcal{M} \backslash Z$.

Proof. We take a Hermitian metric $h_{0}$ of $E$ such that the following holds:

- Each $P \in Z$ has a neighbourhood $\mathcal{U}_{P}$ in $\mathcal{M}$ such that (i) $G\left(h_{0}\right)=0$ on $\mathcal{U}_{P} \backslash\{P\}$, (ii) $P$ is Dirac type singularity of the monopole $\left(E, \bar{\partial}_{E}, h_{0}\right)_{\mid \mathcal{U}_{P} \backslash\{P\}}$.

Let $f$ be any $C^{\infty}$-function on $\mathcal{M}$. Note that $G\left(h_{0} e^{f}\right)-G\left(h_{0}\right)=4^{-1} \Delta f$, where $\Delta$ denote the Laplacian of $\mathcal{M}$. (See [4, Section 2.8.4] for the untwisted case. The twisted case can be argued similarly.) Because

$$
\int_{\mathcal{M}} G\left(h_{0}\right) \frac{\sqrt{-1}}{2} d t d w d \bar{w}=\mu_{B}=-\frac{1}{2} \int_{\mathcal{M}} B^{(1,1)} d t
$$

there exists an $\mathbb{R}$-valued $C^{\infty}$-function $f_{1}$ such that $\left(\Delta f_{1}\right) d w d \bar{w}=-4\left(G\left(h_{0}\right) d w d \bar{w}-\sqrt{-1} B^{(1,1)}\right)$. Then, the claim of Lemma 3.22 follows.

Let us study the case where $\varrho_{B}=0$, which implies $B=B^{(1,1)}$. On $\mathbb{R}^{4}=\mathbb{R} \times \mathbb{R}^{3}$, we use the real coordinate system $(s, t, x, y)$ and the complex coordinate system $(z, w)$ given by $z=s+\sqrt{-1} t$ and $w=x+\sqrt{-1} y$.

Let $\widetilde{\Gamma}$ denote the lattice of $\mathbb{R}^{4}=\mathbb{R} \times(\mathbb{R} \times \mathbb{C})$ generated by $(1,0,0)$ and $\left(0, a_{i}, \alpha_{i}\right)(i=1,2,3)$. We consider the action of $\widetilde{\Gamma}$ on $\mathbb{R}^{4}$ induced by the natural $\mathbb{Z}$-action on $\mathbb{R}$ and the $\Gamma$-action on $\mathbb{R} \times \mathbb{C}$. Let $\left(X, g_{X}\right)$ denote the Kähler manifold obtained as the quotient of $\left(\mathbb{C}^{2}, d z d \bar{z}+d w d \bar{w}\right)$ by the $\widetilde{\Gamma}$-action. Let $p: X \longrightarrow \mathcal{M}$ denote the naturally defined projection.

We set $\widetilde{E}:=p^{-1}(E)$ on $X \backslash p^{-1}(Z)$. It is equipped with the complex structure $\bar{\partial}_{\widetilde{E}}$ determined by

$$
\partial_{\widetilde{E}, \bar{w}} p^{-1}(u)=p^{-1}\left(\partial_{E, \bar{w}} u\right), \quad \partial_{\widetilde{E}, \bar{z}} p^{-1}(u)=\frac{1}{2} \cdot p^{-1}\left(\phi \cdot u+\sqrt{-1} \partial_{E, t} u\right)
$$

for sections $u$ of $E$. For any adapted Hermitian metric $h_{0}$ of $E$, set $\widetilde{h}_{0}:=p^{-1}\left(h_{0}\right)$.

Let $F\left(\widetilde{h}_{0}\right)$ denote the curvature of the Chern connection of $\left(\widetilde{E}, \bar{\partial}_{\widetilde{E}}, \widetilde{h}_{0}\right)$. Let $\Lambda$ denote the contraction from $(1,1)$-forms to $(0,0)$-forms with respect to the Kähler form of $\left(X, g_{X}\right)$. Then, $\sqrt{-1} \Lambda F\left(\widetilde{h}_{0}\right)=p^{-1}\left(G\left(h_{0}\right)\right)$ holds.

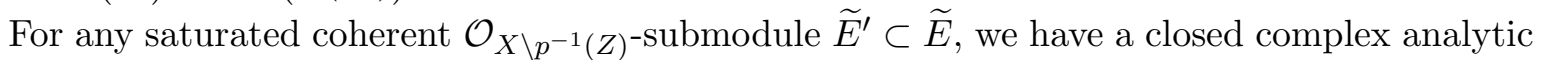
subset $W \subset X \backslash p^{-1}(Z)$ with complex codimension 2 such that $\widetilde{E}^{\prime}$ is a subbundle of $\widetilde{E}$ outside of $W$. We have the induced metric $\widetilde{h}_{0, \widetilde{E}^{\prime}}$ of $\widetilde{E}_{\mid X \backslash\left(p^{-1}(Z) \cup W\right)}^{\prime}$. We define

$$
\operatorname{deg}\left(\widetilde{E}^{\prime}, \widetilde{h}_{0}\right):=\sqrt{-1} \int \operatorname{Tr} \Lambda F\left(\widetilde{h}_{0, \widetilde{E}^{\prime}}\right) \operatorname{dvol}_{X}
$$

Because of the Chern-Weil formula, it is well defined in $\mathbb{R} \cup\{-\infty\}$ as explained in [7]. Then, $\left(\widetilde{E}, \bar{\partial}_{\widetilde{E}}, \widetilde{h}_{0}\right)$ is defined to be analytically stable with respect to the $S^{1}$-action if

$$
\frac{\operatorname{deg}\left(\widetilde{E}^{\prime}, \widetilde{h}_{0}\right)}{\operatorname{rank} \widetilde{E}^{\prime}}<\frac{\operatorname{deg}\left(\widetilde{E}, \widetilde{h}_{0}\right)}{\operatorname{rank} \widetilde{E}}
$$

holds for any $S^{1}$-invariant saturated subsheaf $\widetilde{E}^{\prime} \subset \widetilde{E}$ with $0<\operatorname{rank} \widetilde{E}^{\prime}<\operatorname{rank} \widetilde{E}$. The following is clear. 
Lemma 3.23. $\left(\widetilde{E}, \bar{\partial}_{\widetilde{E}}, \widetilde{h}_{0}\right)$ is analytically stable with respect to the $S^{1}$-action if and only if $\left(E, \bar{\partial}_{E}, h_{0}\right)$ is analytically stable.

According to Lemma 3.22 , there exists a Hermitian metric $h_{\operatorname{det}(E)}$ such that $\left(E, \bar{\partial}_{E}, h_{\operatorname{det}(E)}\right)$ is a $(\operatorname{rank} E) B$-twisted monopole. We take an adapted Hermitian metric $h_{-1}$ such that each $P \in Z$ has a neighbourhood $\mathcal{U}_{P}$ such that $G\left(h_{-1}\right)_{\mid \mathcal{U}_{P} \backslash\{P\}}=0$. An $\mathbb{R}$-valued $C^{\infty}$-function $f$ is determined by $\operatorname{det}\left(h_{-1}\right)=h_{\operatorname{det}(E)} e^{f}$. We set $h_{0}=h_{-1} e^{-f / \operatorname{rank}(E)}$. Then, $h_{0}$ is an adapted metric of $E$. By Lemma $3.23,\left(\widetilde{E}, \bar{\partial}_{\widetilde{E}}, \widetilde{h}_{0}\right)$ is analytically stable with respect to the $S^{1}$-action. We also have $\Lambda \operatorname{Tr} F\left(\widetilde{h}_{0}\right)=\sqrt{-1} \operatorname{rank}(E) p^{-1}(B)$. According to a theorem of Simpson [7, Theorem 1], there exists an $S^{1}$-invariant metric $\widetilde{h}$ of $\widetilde{E}$ such that (i) $\operatorname{det}(\widetilde{h})=\operatorname{det}\left(\widetilde{h}_{0}\right)$, (ii) $\Lambda F(\widetilde{h})=$ $\sqrt{-1} p^{-1}(B) \mathrm{id}_{\widetilde{E}}$, (iii) $\widetilde{h}$ and $\widetilde{h}_{0}$ are mutually bounded. We obtain the corresponding metric $h$ of $E$, for which $G(h)=\sqrt{-1} B$ id $E$ holds. Because $h$ and $h_{0}$ are mutually bounded, each $P \in Z$ is a Dirac type singularity of $\left(E, \bar{\partial}_{E}, h\right)$ which is implied by [6, Theorem 3]. Thus, we obtain the claim of Proposition 3.21 in the case $\varrho_{B}=0$.

Let us study the case where $\varrho_{B}$ is not necessarily 0 .

Lemma 3.24. There exist a finite subset $Z_{1} \subset \mathcal{M}$ and $a \varrho_{B}$-twisted mini-holomorphic bundle $\left(E_{1}, \bar{\partial}_{E_{1}}\right)$ with Dirac type singularity of rank one on $\left(\mathcal{M} ; Z_{1}\right)$ such that $\operatorname{deg}^{\text {an }}\left(E_{1}, \bar{\partial}_{E_{1}}\right)=\mu_{B}$.

Proof. It follows from Lemma 2.18 and Proposition 3.13.

We set $\left(E^{\prime}, \bar{\partial}_{E^{\prime}}\right):=\left(E, \bar{\partial}_{E}\right) \otimes\left(E_{1}, \bar{\partial}_{E_{1}}\right)^{-1}$. Then, $\left(E^{\prime}, \bar{\partial}_{E^{\prime}}\right)$ is a stable mini-holomorphic bundle with $\mu^{\text {an }}\left(E^{\prime}, \bar{\partial}_{E^{\prime}}\right)=0$. According to the claim in the case $\varrho_{B}=0$, there exists an adapted Hermitian metric $h^{\prime}$ of $\left(E^{\prime}, \bar{\partial}_{E^{\prime}}\right)$ such that $\left(E^{\prime}, \bar{\partial}_{E^{\prime}}, h^{\prime}\right)$ is a monopole. According to Lemma 3.22, there exists a Hermitian metric $h_{1}$ of $E_{1}$ such that $\left(E_{1}, \bar{\partial}_{E_{1}}, h_{1}\right)$ is a $B$-twisted monopole with Dirac type singularity. Let $h$ be the Hermitian metric of $E$ induced by $h^{\prime}$ and $h_{1}$. Then, $h$ is adapted to $\left(E, \bar{\partial}_{E}\right)$, and $\left(E, \bar{\partial}_{E}, h\right)$ is a $B$-twisted monopole. Thus the proof of Proposition 3.21 is completed.

\section{A more sophisticated formulation of the stability condition}

We explain that the analytic stability condition (Definition 3.9) is equivalent to the stability condition introduced by Kontsevich and Soibelman in the case $\varrho=0$ (see Section 1.2). This section is devoted to explain their idea of degree.

\subsection{Preliminary}

\subsubsection{Closed 1-forms and 1-homology}

Let $A$ be a 3 -dimensional manifold. Let $Z_{\mathrm{DR}}^{i}(A)$ denote the space of closed $i$-form $\tau$ on $A$. Let $B$ be finite subset of $A$. Let $H_{j}(A, B)$ denote the relative $j$-th homology group with $\mathbb{R}$-coefficient.

Let $\gamma$ be any element of $H_{1}(A, B)$. We take a representative of $\gamma$ by a smooth 1 -chain $\widetilde{\gamma}$. For any $\omega \in Z_{\mathrm{DR}}^{1}(A)$, the number $\int_{\widetilde{\gamma}} \omega$ is independent of the choice of a representative $\widetilde{\gamma}$. They are denoted by $\int_{\gamma} \omega$.

Let $C^{\infty}(A, B)$ denote the space of $C^{\infty}$-functions $f$ on $A$ such that $f(P)=0$ for any $P \in B$. Let $Z_{\mathrm{DR}}^{1}(A)$ denote the space of closed 1-forms on $A$. Let $B_{\mathrm{DR}}^{1}(A, B)$ denote the image of $d: C^{\infty}(A, B) \longrightarrow Z_{\mathrm{DR}}^{1}(A)$. Because $\int_{\gamma} d f=0$ for any $f \in C^{\infty}(A, B)$, we obtain the well defined map

$$
\int_{\gamma}: Z_{\mathrm{DR}}^{1}(A) / B_{\mathrm{DR}}^{1}(A, B) \longrightarrow \mathbb{R} .
$$




\subsubsection{Duality}

Suppose that $A$ is compact and oriented. Let $H^{j}(A \backslash B)$ denote the $j$-th de Rham cohomology group of $A \backslash B$. Let $H_{c}^{j}(A \backslash B)$ denote the $j$-th de Rham cohomology group with compact support. We have the non-degenerate pairing between $H^{2}(A \backslash B)$ and $H_{c}^{1}(A \backslash B)$ induced by the cup product and the integration. We also have the non-degenerate pairing between $H_{c}^{1}(A \backslash B)$ and $H_{1}(A, B)$ induced by the integration. Hence, we obtain the isomorphism

$$
\Phi_{A, B}: H^{2}(A \backslash B) \simeq H_{1}(A, B) .
$$

By definition, for any $a \in H^{2}(A \backslash B)$ and $b \in H_{c}^{1}(A \backslash B)$, the following holds:

$$
\int_{\Phi_{A, B}(a)} b=\int_{A} a \wedge b
$$

Take any Riemannian metric $g_{A}$ of $A$. For any $j$-form $\tau$ on $A \backslash B$, let $|\tau|_{g_{A}}$ denote the function on $A \backslash B$ obtained as the norm of $\tau$ with respect to $g_{A}$.

Lemma 4.1. Let $\tau \in Z_{\mathrm{DR}}^{2}(A \backslash B)$ such that $|\tau|_{g_{A}}$ is an $L^{1}$-function on $A$. Then, the following holds for any $\rho \in Z_{\mathrm{DR}}^{1}(A)$ :

$$
\int_{\Phi_{A, B}([\tau])} \rho=\int_{A} \rho \wedge \tau
$$

Here, $[\tau] \in H_{\mathrm{DR}}^{2}(A \backslash B)$ denotes the cohomology class of $\tau$.

Proof. For any point $P \in Z$, we take a small coordinate neighbourhood $\left(A_{P}, x_{P, 1}, x_{P, 2}, x_{P, 3}\right)$ of $P$ such that (i) $P$ corresponds to $(0,0,0)$, (ii) $A_{P} \simeq\left\{\left(x_{1}, x_{2}, x_{3}\right) \in \mathbb{R}^{3} \mid \sum x_{i}^{2}<1\right\}$ by the coordinate system. Set $\left\|\boldsymbol{x}_{P}\right\|:=\left(x_{P, 1}^{2}+x_{P, 2}^{2}+x_{P, 3}^{2}\right)^{1 / 2}$. Then, there exists a $C^{\infty}$-function $f_{P}$ on $A_{P}$ such that (i) $d f_{P}=\rho$ on $\left\{\left\|\boldsymbol{x}_{P}\right\|<1 / 2\right\}$, (ii) $f_{P}(P)=0$, (iii) $f_{P}(Q)=0$ for $Q \in\left\{\left\|\boldsymbol{x}_{P}\right\|>\right.$ $2 / 3$. We naturally regard $f_{P}$ as a $C^{\infty}$-function on $A$. Then, the following holds:

$$
\begin{aligned}
\int_{\Phi_{A, B}([\tau])} \rho & =\int_{\Phi_{A, B}([\tau])}\left(\rho-\sum_{P \in B} d f_{P}\right) \\
& =\int_{A}\left(\rho-\sum_{P \in B} d f_{P}\right) \wedge \tau=\int_{A} \rho \wedge \tau-\sum_{P} \int_{A} d\left(f_{P} \tau\right) .
\end{aligned}
$$

For each $P$, we set $S_{P}^{2}(r):=\left\{\left\|\boldsymbol{x}_{P}\right\|=r\right\}$ with the orientation as the boundary of $\left\{\left\|\boldsymbol{x}_{P}\right\| \leq r\right\}$. Then, we obtain the following

$$
\int_{A} d\left(f_{P} \tau\right)=-\lim _{\epsilon \rightarrow 0} \int_{S_{P}^{2}(\epsilon)} f_{P} \tau
$$

Note that the limit exists because $d\left(f_{P} \tau\right)=d f_{P} \wedge \tau$ is integrable. Because $|\tau|_{g_{A}}$ is $L^{1}$, we have $\int d r \int_{S_{P}^{2}(r)}|\tau|_{g_{A}}<\infty$, and hence there exists a sequence $r_{i} \rightarrow 0$ such that $r_{i} \int_{S_{P}^{2}\left(r_{i}\right)}|\tau|_{g_{A}} \rightarrow 0$. Because $\left|f_{P}\right|=O\left(\left\|\boldsymbol{x}_{P}\right\|\right)$, we obtain that (4.1) is 0 .

\subsection{Relation between degrees of mini-holomorphic bundles}

Let $\mathcal{M}$ be as in Section 3. We may naturally regard $\mathcal{M}$ as a 3 -dimensional abelian Lie group. Let $\mathfrak{T}$ denote the space of the invariant vector fields on $\mathcal{M}$. Let $\mathfrak{T}^{\vee}$ denote the space of the invariant 1 -forms on $\mathcal{M}$. We have the natural non-degenerate paring $\mathfrak{T} \otimes \mathfrak{T}^{\vee} \longrightarrow \mathbb{R}$. We have the dual morphism $\mathbb{R} \longrightarrow \mathfrak{T}^{\vee} \otimes \mathfrak{T}$. Let $\sigma$ denote the image of 1 . If we take a base $e_{i}(i=1,2,3)$ 
of $\mathfrak{T}$ and the dual frame $e_{i}^{\vee}(i=1,2,3)$, then $\sigma=\sum e_{i}^{\vee} \otimes e_{i}$. For the mini-complex coordinate $(t, w)$, we have $\sigma=d t \otimes \partial_{t}+d w \otimes \partial_{w}+d \bar{w} \otimes \partial_{\bar{w}}$.

Let $E$ be a vector bundle on $\mathcal{M} \backslash Z$. Kontsevich and Soibelman [2] introduced the following element:

$$
\int_{\Phi_{\mathcal{M}, Z}\left(c_{1}(E)\right)} \sigma \in \mathfrak{T}
$$

Proposition 4.2. Let $\varrho=\varrho_{0} d t d \bar{w}$ be a 2-form on $\mathcal{M}$. Let $\left(E, \bar{\partial}_{E}\right)$ be a $\varrho$-twisted miniholomorphic bundle with Dirac type singularity on $(\mathcal{M} ; Z)$. Then,

$$
\int_{\Phi_{\mathcal{M}, Z}\left(c_{1}(E)\right)} \sigma=\frac{1}{\pi} \operatorname{deg}^{\operatorname{an}}(E) \cdot \partial_{t}-\frac{\operatorname{rank}(E)}{\pi}\left(\left(\int_{\mathcal{M}} \varrho_{0}\right) \partial_{w}+\left(\int_{\mathcal{M}} \overline{\varrho_{0}}\right) \partial_{\bar{w}}\right) .
$$

In particular, if $\varrho=0$, then the following holds:

$$
\int_{\Phi_{\mathcal{M}, Z}\left(c_{1}(E)\right)} \sigma=\frac{1}{\pi} \operatorname{deg}^{\mathrm{an}}(E) \cdot \partial_{t} .
$$

Proof. Let $h$ be an adapted metric of $\left(E, \bar{\partial}_{E}\right)$. By Lemma 4.1, it is enough to prove the following equality:

$$
\begin{aligned}
\frac{\sqrt{-1}}{2} \int_{\mathcal{M}} \operatorname{Tr} F(h) \cdot \sigma= & \int_{\mathcal{M}} \operatorname{Tr} G(h) \operatorname{dvol}_{\mathcal{M}} \cdot \partial_{t} \\
& -\operatorname{rank}(E)\left(\left(\int_{\mathcal{M}} \varrho_{0}\right) \partial_{w}+\left(\int_{\mathcal{M}} \overline{\varrho_{0}}\right) \partial_{\bar{w}}\right) .
\end{aligned}
$$

For $\kappa=t, w, \bar{w}$, we obtain the following by the Stokes formula and the estimate $\left|\phi_{h, Q}\right|_{h}=$ $O\left(d(P, Q)^{-1}\right):$

$$
\int \operatorname{Tr}\left(\nabla_{h, \kappa} \phi_{h}\right) \frac{\sqrt{-1}}{2} d t d w d \bar{w}=0
$$

Note that $F(h)_{t \bar{w}}+\sqrt{-1} \nabla_{\bar{w}} \phi=\varrho_{0} \operatorname{id}_{E}$ and $F(h)_{t w}-\sqrt{-1} \nabla_{w} \phi=-\varrho_{0} \mathrm{id}_{E}$, according to Lemma 2.12. We obtain

$$
\begin{aligned}
\frac{\sqrt{-1}}{2} \int \operatorname{Tr} F(h) d w \otimes \partial_{w} & =\int \operatorname{Tr}\left(F(h)_{t \bar{w}}+\sqrt{-1} \nabla_{\bar{w}} \phi\right) \frac{\sqrt{-1}}{2} d t d \bar{w} d w \otimes \partial_{w} \\
& =-\operatorname{rank}(E)\left(\int_{\mathcal{M}} \varrho_{0}\right) \partial_{w} .
\end{aligned}
$$

Similarly, we obtain

$$
\frac{\sqrt{-1}}{2} \int \operatorname{Tr} F(h) d \bar{w} \otimes \partial_{\bar{w}}=-\operatorname{rank}(E)\left(\int_{\mathcal{M}} \overline{\varrho_{0}}\right) \partial_{\bar{w}} .
$$

We also obtain the following from (4.3):

$$
\begin{aligned}
\frac{\sqrt{-1}}{2} \int \operatorname{Tr} F(h)_{w \bar{w}} d w d \bar{w} d t \otimes \partial_{t} & =\int \operatorname{Tr}\left(F(h)_{w \bar{w}}-\frac{\sqrt{-1}}{2} \nabla_{h, t} \phi_{h}\right) \frac{\sqrt{-1}}{2} d w d \bar{w} d t \otimes \partial_{t} \\
& =\left(\int_{\mathcal{M}} \operatorname{Tr} G(h)\right) \partial_{t} .
\end{aligned}
$$

Thus, we obtain (4.2), and the proof of Proposition 4.2 is completed.

Remark 4.3. As explained in Section 1.2, Kontsevich and Soibelman [2] formulated the stability condition for mini-holomorphic bundles in terms of the coefficient of $\partial_{t}$ in $\int_{\Phi_{Z}\left(c_{1}(E)\right)} \sigma$. 


\section{Acknowledgements}

I thanks Maxim Kontsevich and Yan Soibelman for the communications and for sending the preprint [2]. Indeed, this study grew out of my answer to one of their questions. They also kindly suggested that there should be a generalization to the twisted case. I hope that this would be useful for their big project. I owe much to Carlos Simpson whose ideas on the Kobayashi-Hitchin correspondence are fundamental in this study. I thank Masaki Yoshino for discussions. I thank the referees for their careful readings and valuable comments.

I am grateful to the organizers of the conference "Integrability, Geometry and Moduli" to celebrate 60 th birthday of Motohico Mulase. The twisted version of the equivalences was explained in my talk at the conference.

It is my great pleasure to dedicate this paper to Motohico Mulase with appreciation to his friendly encouragements and suggestions on many occasions.

I am partially supported by the Grant-in-Aid for Scientific Research (S) (No. 17H06127), the Grant-in-Aid for Scientific Research (S) (No. 16H06335), and the Grant-in-Aid for Scientific Research (C) (No. 15K04843), Grant-in-Aid for Scientific Research (C) (No. 20K03609), Japan Society for the Promotion of Science.

\section{References}

[1] Charbonneau B., Hurtubise J., Singular Hermitian-Einstein monopoles on the product of a circle and a Riemann surface, Int. Math. Res. Not. 2011 (2011), 175-216, arXiv:0812.0221.

[2] Kontsevich M., Soibelman Y., Riemann-Hilbert correspondence in dimension one, Fukaya categories and periodic monopoles, Preprint.

[3] Kronheimer P.B., Monopoles and Taub-NUT metrics, Master Thesis, Oxford, 1986.

[4] Mochizuki T., Periodic monopoles and difference modules, arXiv:1712.08981.

[5] Mochizuki T., Doubly-periodic monopoles and $q$-difference modules, arXiv:1902.08298.

[6] Mochizuki T., Yoshino M., Some characterizations of Dirac type singularity of monopoles, Comm. Math. Phys. 356 (2017), 613-625, arXiv:1702.06268.

[7] Simpson C.T., Constructing variations of Hodge structure using Yang-Mills theory and applications to uniformization, J. Amer. Math. Soc. 1 (1988), 867-918.

[8] Yoshino M., A Kobayashi-Hitchin correspondence between Dirac-type singular mini-holomorphic bundles and HE-monopoles, arXiv:1902.09995. 